\title{
Multi-Objective Techno-Economic-Environmental Optimisation of Electric Vehicle for Energy Services
}

\author{
Ridoy Das ${ }^{\mathrm{a} 1}$, Yue Wang ${ }^{\mathrm{b}}$, Ghanim Putrus ${ }^{\mathrm{a}}$, Richard Kotter ${ }^{\mathrm{a}}$, Mousa Marzband ${ }^{\mathrm{a}}$, Bert Herteleer ${ }^{\mathrm{c}}$ and \\ Jos Warmerdam ${ }^{\text {d }}$ \\ ${ }^{a}$ Northumbria University, Electrical Power and Control System Research Group, 2 Ellison Place NE1 8ST, \\ Newcastle upon Tyne, United Kingdom \\ ${ }^{\mathrm{b}}$ University of Chichester, Engineering and Design, College Lane, PO19 6PE, Chichester, United Kingdom \\ ' KU Leuven, Technology cluster Electrical Engineering (ESAT), De Smetstraat brothers 1, 9000 Ghent, \\ Belgium \\ d Amsterdam University of Applied Sciences, Amstelcampus, Weesperzijde 190, 1097 DZ Amsterdam, \\ Netherlands
}

\begin{abstract}
Electric vehicles and renewable energy sources are collectively being developed as a synergetic implementation for smart grids. In this context, smart charging of electric vehicles and vehicle-to-grid technologies are seen as a way forward to achieve economic, technical and environmental benefits. The implementation of these technologies requires the cooperation of the end-electricity user, the electric vehicle owner, the system operator and policy makers. These stakeholders pursue different and sometime conflicting objectives. In this paper, the concept of multi-objective-techno-economicenvironmental optimisation is proposed for scheduling electric vehicle charging/discharging. End user energy cost, battery degradation, grid interaction and $\mathrm{CO}_{2}$ emissions in the home micro-grid context are modelled and concurrently optimised for the first time while providing frequency regulation. The results from three case studies show that the proposed method reduces the energy cost, battery degradation, $\mathrm{CO}_{2}$ emissions and grid utilisation by $88.2 \%, 67 \%, 34 \%$ and $90 \%$ respectively, when compared to uncontrolled electric vehicle charging. Furthermore, with multiple optimal solutions, in order to achieve a $41.8 \%$ improvement in grid utilisation, the system operator needs to compensate the end electricity user and the electric vehicle owner for their incurred benefit loss of $27.34 \%$ and $9.7 \%$ respectively, to stimulate participation in energy services.
\end{abstract}

\section{Highlights}

- Optimisation of energy cost, battery degradation, grid utilisation and $\mathrm{CO}_{2}$ emission

- The conflicts among objectives were addressed with multi-objective optimisation

- A multi-criteria decision making process was tailored to the stakeholders

\footnotetext{
${ }^{1}$ Corresponding author: ridoy.das@ @orthumbria.ac.uk, Northumbria University, Department of Mathematics Physics and Electrical Engineering, Ellison Place NE1 8ST, Newcastle upon Tyne, United Kingdom.
} 
- Frequency regulation provision was overall profitable for electric vehicle owners

- The decision makers must cooperate to achieve societal benefits

\begin{tabular}{|c|c|}
\hline \multicolumn{2}{|c|}{ Nomenclature } \\
\hline $\begin{array}{l}\text { Acronyms } \\
\text { AHP }\end{array}$ & Analytical hierarchy process \\
\hline ANEC & $\begin{array}{l}\text { Augmented non-dominated } \varepsilon \text {-constraint } \\
\text { method }\end{array}$ \\
\hline $\mathrm{DM}$ & Decision maker \\
\hline $\mathrm{DN}$ & Distribution network \\
\hline DOD & Depth of discharge \\
\hline DSO & Distribution system operator \\
\hline EOL & End of life \\
\hline EV & Electric vehicle \\
\hline FFR & Firm frequency response \\
\hline ICE & Internal combustion engine \\
\hline LV & Low Voltage \\
\hline MCDM & Multi-criteria-decision-making \\
\hline MOO & Multi-objective optimisation \\
\hline MOTEEO & $\begin{array}{l}\text { Multi-objective techno-economic- } \\
\text { environmental optimisation }\end{array}$ \\
\hline PV & Photovoltaic \\
\hline RES & Renewable energy source \\
\hline SOC & State of charge \\
\hline TSO & Transmission system operator \\
\hline V2G & Vehicle-to-grid \\
\hline $\mathrm{V} 2 \mathrm{H}$ & Vehicle-to-home \\
\hline \multicolumn{2}{|c|}{ Sets and indices } \\
\hline$t$ & Current time step \\
\hline$\Delta t$ & Time interval, 15 min. \\
\hline$N^{s}$ & Total number of simulated time steps \\
\hline$N^{a}$ & $\begin{array}{l}\text { Total number of steps from arrival to } \\
\text { departure }\end{array}$ \\
\hline \multicolumn{2}{|l|}{ Constants } \\
\hline$\frac{\eta}{F^{E V}}$ & $\begin{array}{l}\text { Efficiency of the EV charger } \\
\text { EV Battery canacity }(\mathrm{kWh})\end{array}$ \\
\hline$\underline{E^{E V}}$ & $\begin{array}{l}\text { Minimum EV battery capacity limit } \\
(\mathrm{kWh})\end{array}$ \\
\hline $\bar{f}$ & $\begin{array}{l}\text { Upper frequency limit of droop- } \\
\text { controller }(\mathrm{Hz})\end{array}$ \\
\hline$\underline{f}$ & $\begin{array}{l}\text { Lower frequency limit of the droop- } \\
\text { controller }(\mathrm{Hz})\end{array}$ \\
\hline$\overline{P^{E V}}$ & $\begin{array}{l}\text { Maximum charging/discharging rate of } \\
\text { the EV charger }(\mathrm{kW})\end{array}$ \\
\hline \multicolumn{2}{|l|}{ Parameters } \\
\hline $\begin{array}{l}\pi_{t} \\
p^{d}\end{array}$ & Real-time price signal at time $\mathrm{t}(\mathfrak{f} / \mathrm{kWh})$ \\
\hline $\begin{array}{l}P_{t}^{d} \\
P_{t}^{P V}\end{array}$ & $\begin{array}{l}\text { Electricity demand at time } \mathrm{t}(\mathrm{kW}) \\
\text { PV generation at time } \mathrm{t}(\mathrm{kW})\end{array}$ \\
\hline
\end{tabular}




\begin{tabular}{|ll|}
\hline$e_{t}^{k W h}$ & Specific $\mathrm{CO}_{2}$ emission $\left(\mathrm{kgCO}_{2} / \mathrm{kWh}\right)$ \\
$C^{B}$ & Cost of the battery $(£ / \mathrm{kWh})$ \\
$T^{B}$ & Temperature of the battery $\left(^{\circ}\right)$ \\
$\beta_{1 \rightarrow 8}$ & Fitting parameters of the battery \\
& degradation model \\
$r g^{t}$ & Regulation signal for FFR $(\mathrm{kW})$ \\
$f$ & Electrical frequency $(\mathrm{Hz})$ \\
$k^{d}$ & Droop coefficient $(\mathrm{kW} / \mathrm{Hz})$ \\
$t^{a}$ & Arrival time of the EV \\
$t^{d}$ & Departure time of the EV \\
$A_{t}^{E V}$ & Availability of the EV at time $\mathrm{t}$ \\
$E^{E V, a}$ & Energy of the EV upon arrival $(\mathrm{kWh})$ \\
$E^{t r i p}$ & Energy required for the next trip $(\mathrm{kWh})$ \\
& \\
$\mathrm{Functions}^{e}$ & \\
$\mathbb{C}^{e}$ & Energy cost of a H-MG $(£ / \mathrm{kWh})$ \\
$\mathbb{C}^{d}$ & Battery degradation cost $(£ / \mathrm{kWh})$ \\
$\mathbb{P}^{G}$ & Grid net exchange $(\mathrm{kWh})$ \\
$\mathbb{E}^{C O 2}$ & CO ${ }_{2}$ emissions of the $\left.\mathrm{H}-\mathrm{MG}(\mathrm{kgCO})_{2}\right)$ \\
$E_{t}^{E V}$ & Energy of the EV at time $\mathrm{t}(\mathrm{kWh})$ \\
$E_{t}^{L}$ & Lifetime energy throughput under a \\
$n^{E O L}$ & certain charging condition $(\mathrm{kWh})$ \\
$\alpha^{c}$ & Number of cycles before battery EOL \\
$P_{t}^{E V+}, P_{t}^{E V-}$ & Battery degradation coefficient \\
&
\end{tabular}

\section{Introduction}

The uptake of electric vehicles (EVs) is rapidly increasing around the world with the concurrent development in available renewable energy sources (RES) [1]. In this context, a number of stakeholders can achieve benefits; these are the final electricity consumer, the EV owner, the electricity system operators and the policy makers, which collectively will be referred hereby as 'stakeholders'. Smart charging and Vehicle-to-Grid (V2G) are implemented to achieve electricity cost reduction, EV battery degradation minimization, $\mathrm{CO}_{2}$ emission reduction and grid impact optimization. Due to the inherent conflict of the aforementioned dimensions, in order to achieve benefits along one dimension, others must be traded off. In this context, the application of multi-objective optimization (MOO) methods is crucial. The different objectives of the optimization should be modelled based on the goals the stakeholders, who then take a decision on the most suitable EV utilization. In this paper, the concept of multi-objective-techno-economic-environmental optimisation (MOTEEO) is proposed by connecting the stakeholders involved in smart grids with optimal control of EVs. With this innovative concept, EV charging/discharging is controlled to simultaneously optimize technical, economic and environmental benefits.

Several studies have addressed optimal charging scheduling for single objectives and multiple objectives. The grid impact has been widely addressed or identified as a critical objective. In [3], the 
power flow in a distribution network RES was optimally controlled by scheduling EV charging. In [4], Voltage deviation caused by excessive PV generation was successfully mitigated by discharging the batteries of EV fleets. In [5], the electricity demand of a residential building was satisfied with a combination of PV system and EVs. The availability of EVs and their capacity to provide demand peak shaving was investigated in [6]. In the framework proposed in [7], by making use of electricity demand and PV generations forecasts, an aggregator and several EV agents performed load levelling. A decentralised optimisation process for EV charging scheduling was proposed in [8]. Although this work did not consider RES, the proposed method effectively performed load-levelling with a fleet of EVs. A decentralised approach was also proposed in [9] to optimally charge EVs in order to reduce demand peak and variance. Power imbalance reduction was addressed in an optimisation process performed in [10]. This proposed method reduced the mismatch between PV generation and electricity demand. A number of studies also aimed at minimizing the EV charging cost [2] and energy arbitrage [11]. In [12], EV charging/discharging was controlled to implement optimal energy management in a micro-grid with availability of wind generation. This method reduced the energy cost of the building. Provision of frequency regulation was explored in [3]. However, a major lack of research on battery degradation minimization has been identified, as none of the aforementioned studies addressed this issue. Indeed, only [2], [3] and [11] included battery degradation in their economic analysis but only as a constant parameter, based on estimated cycle life and unaffected by the charging schedule. Moreover, apart from [17], the other studies implementing single-objective optimisation did not minimise $\mathrm{CO}_{2}$ emissions.

Only a number of studies in recent literature have applied MOO in the context of smart grids and EVs. In [13] grid load variance was optimised while providing Voltage control by scheduling grid-connected EVs with a centralised approach. However, the objective functions were sequentially optimised and, since the results of the top layer fed the lower layer, the objectives did not conflict with each other. In the approach proposed in the present paper, the critical objective of battery degradation is modelled and optimised whilst ensuring that the transportation requirements of the individual EV are satisfied. This is possible only with a decentralised approach and a multi-objective optimisation as proposed in this paper. Optimal deployment of charging infrastructure to minimise annual investment cost and maximise annual captured traffic flow was proposed in [14]. However, the proposed approach performed a centralised decision plan and did not provide optimal charging scheduling, which would affect the individual EV owner. In [15], EV battery swapping stations were simulated in a distribution network in order to minimize battery charging cost, power loss cost, to flatten the network Voltage profile and release network capacity. Although this study consider the important aspect related to grid constraints, they do not take into account the interests of the EV owner as done in this paper by minimising battery degradation, and do not address environmental objectives as we do with $\mathrm{CO}_{2}$ emission reduction. In [16], optimal scheduling of energy storage systems by minimising both battery calendar degradation and energy costs was implemented. However, in their proposed methodology, the two objectives were 
linearly combined. Such a method is not feasible for other objectives such as load levelling because appropriate, and often unknown, weights must be assigned. Furthermore, this approach does not present a Pareto analysis, as we do in our proposed framework, which is essential for multi-criteria-decision making where several objectives are involved. In [17], a notable approach of scheduling EV charging to minimize cost and emission was proposed, which accounted for two out of the four objectives considered in our work. However, their proposed method did not consider battery degradation nor the impact of EV charging on the grid. In [18] an improved optimal power flow in a distribution network with EVs, wind energy and PV was implemented to address $\mathrm{CO}_{2}$ emission and operational cost. The uncertainty regarding RES generation and EV availability considered with a Monte Carlo simulation and multi-objective genetic algorithm was implemented to address the two objectives. This study was able to highlight the trade-off between the two objectives, however, as the authors themselves point out, their centralised approach suffers from high computational expense, at the point that parallel computation was proposed as a solution to reduce this burden. Furthermore, battery degradation was not addressed in their work. Fuel consumption and battery degradation and were linearly combined for optimal drive-train energy management strategy in [19]. Although the approach proposed in the paper is effective in optimising the two objectives, the interaction with the grid was not investigated since no charging scheduling was implemented. Load variance and charging cost were minimised with a weighted sum method in [20] with a decentralised approach. Although some measures to reduce battery degradation were mentioned, i.e. reduce the maximum SOC level, it was not optimised as a separate objective. Similarly no mention was made on the environmental footprint of the charging process, and the weighted sum method may not find Pareto solutions if the final objective function is not convex; in general $\varepsilon$-constraint is superior as it overcomes such problem

Game theory based approaches have also been implemented to energy management (see [21]). However, under MOTEEO every charging schedule leads to different outcomes (modelled by different objective functions), and we propose a framework for decision making that agrees on a single schedule in a set of equally efficient schedules, which constitute a Pareto frontier. This is different from game theory, where different players with different strategies seek a Nash equilibrium.

As evidenced by the literature review, the main research gaps can be summarised as:

- Lack of a holistic solution to simultaneously optimize the critical objectives of energy cost, battery degradation, grid net exchange and $\mathrm{CO}_{2}$ emissions. None of the studies have addressed the tradeoff between these objectives. Indeed, only a subset of the aforementioned objectives has been optimised.

- There is an evident lack of studies addressing battery degradation minimisation as an optimisation process applied to EV charging. 
- $\mathrm{CO}_{2}$ emission has been seldom addressed as an objective, and its conflict with other objectives has not been satisfactorily highlighted.

- The trade-off between ancillary service provision and other energy services has not been explicated in the literature.

- A decision making process tailored to key smart grid stakeholders, namely end electricity user, EV owner and electricity system operator has not been proposed in previous works.

Therefore, the purpose of the present work is to address this research gap and the key contributions may be summarised as follows:

- Simultaneous optimisation of electricity cost, battery degradation, grid net exchange and $\mathrm{CO} 2$ emissions has been performed.

- A dynamic battery model (using empirical data), depicting the impact of three key stress-factors, has been implemented in order to minimize cycle degradation as a key objective.

- The use of EV batteries to provide ancillary services to the grid has been considered as an additional objective and its implications on other objectives has been investigated.

- The conflict of interest among the end electricity user, the EV owner and the system operator has been highlighted and addressed using analytical hierarchy process (AHP) and utility function.

The remainder of the paper is organised as follows: in Section 2, the MOTEEO problem is formulated in accordance with the priorities of five stakeholders. Section 3 details the analytical formulation of the four objectives of the involved stakeholders and ancillary services along with the constraints of the EV model, the MOO and multi-criteria decision-making (MCDM) approach. Section 4 elaborates on the case studies, presenting and discussing the achieved results whilst providing advice to decision makers. In Section 5 the conclusions on the implementation of MOTEEO and its advantages are drawn.

\section{Problem statement}

The implementation of smart grids brings together several stakeholders at different scales. From the consumer-facing level to higher ones the relevant stakeholders are the EV owner, the end electricity user (also owning the PV system and the household electricity appliances), aggregators, distribution system operator (DSO), transmission system operator (TSO) and regulatory bodies - with the latter enforcing environmental targets. Consequently, a variety of stakeholders, which would not necessarily collaborate, are brought together and each of them have their own aims/objectives. Some of these are aligned, whereas in some cases the objectives from the different stakeholders may be in conflict.

In this paper we propose a decentralised optimisation framework for day-ahead EV charging/discharging scheduling, where the information is gathered locally and processed by the individual agents that are in charge of the single home-micro-grids (H-MG). This choice is motivated by the onerous communication network, data privacy and safety issues entailed by a centralised approach [22] (also see [18]). Furthermore, the proposed approach facilitates the scalability of the 
optimisation algorithm with high EV penetration, where the computational burden is shared and not concentrated as in centralised management frameworks. The approach proposed in this paper is particularly suitable for peer-to-peer energy trading [23], as each agent maximises the benefit of the respective user and with a local energy market in place, the users could trade energy under regulated price signals.

We define EV charging/discharging strategies and services, to benefit a variety of stakeholders including smart charging, V2X (vehicle to archetype), smart grid services, such as energy arbitrage and ancillary services (e.g. frequency response). The services that we consider ranging from the transmission level, to services behind the meter are: ancillary services (involving the TSO), peak shaving, (involving the DSO), energy bill reduction and energy-autonomy maximisation, both involving the end-electricity user and the policy-maker (since increased energy autonomy achieves emission reduction).

As EV batteries are costly, utilizing them for the aforementioned services may cause additional battery wear. We safeguard the EV owner by minimizing battery degradation with an empirical dynamic model. The aforementioned services are provided by considering transportation as the main purpose for EVs, therefore this is taken as a constraint in the EV model. The proposed framework prioritises the inviolable EV travelling requirements, hence the charging scheduling are always compatible with the EV owner's need. From the end user to the DSO, the objectives modelled in this work are:

- $O b j_{1}$ is the energy cost of the dwelling, which is modelled based on a real-time price and taking into account the local PV generation.

- $\mathrm{Obj}_{2}$ is the battery degradation incurred for EV charging/discharging both for transportation requirement and energy services.

- $\mathrm{Obj}_{3}$ is the grid net exchange, which account for the interaction of the power absorbed/injected by the dwelling from/to the grid.

- $\mathrm{Obj}_{4}$ is the $\mathrm{CO}_{2}$ emission caused by absorbing energy from the grid.

It should be pointed out that although the present work models four objectives, as will be explained in Section 4.1, under suitable assumptions, $\mathrm{Obj}_{3}$ and $\mathrm{Obj}_{4}$ are comparable. Hence, in the proposed methodology, $O b j_{1}, O b j_{2}$ and $O b j_{3}$ will be optimised.

Another critical stakeholder is the TSO, who procures ancillary services to ensure stable operation of the transmission network. As transmission and distribution networks are connected, the TSO is also considered here as a stakeholder. Therefore, ancillary service provision is modelled in the current work as an additional scenario; more details are provided in section 3.5.

We then propose a multi-objective techno-economic-environmental optimisation (MOTEEO) framework and apply it to three case studies with two scenarios to provide the stakeholders with a 
comprehensive assessment of the prospective benefits. Table 1 outlines the case studies and the scenarios simulated in the current work.

Table 1 Case study and scenarios for MOTEEO

\begin{tabular}{lll}
\hline & $\begin{array}{l}\text { Scenario } \text { i) without ancillary } \\
\text { service }\end{array}$ & $\begin{array}{l}\text { Scenario ii) with ancillary } \\
\text { service }\end{array}$ \\
\hline $\begin{array}{l}\text { Case study 1: home-micro-grid } \\
\text { (H-MG) }\end{array}$ & Bidirectional home charging & Bidirectional home charging \\
\hline Case study 2: distribution grid & $\begin{array}{l}\text { a) Uncontrolled charging } \\
\text { b) Smart charging } \\
\text { c) Bidirectional home charging } \\
\text { d) Bidirectional home and } \\
\text { work charging }\end{array}$ & e) Bidirectional home charging \\
& \multicolumn{1}{c}{ Bidirectional home charging } \\
\hline $\begin{array}{l}\text { Case 3: utility function in } \\
\text { home-micro-grid }\end{array}$ & & \\
\hline
\end{tabular}

In the first case study, we highlight the conflicts among the objectives of the stakeholders (and ancillary service), and implement MOTEEO to a single dwelling with one EV. We evaluate two scenarios, aiming to show the additional benefits of ancillary service provision. To quantify the benefits on a higher level, i.e. for the DSO, we then apply MOTEEO to an electricity distribution system with multiple dwellings and EVs. Smart charging and bidirectional charging strategies are applied in home and workplaces. In the case $2 \mathrm{~d}$, the EV can be charged at the workplace where we assume a PV system is present. Finally, we consider case study 3 , where the utility function can be applied to combine the energy cost, battery degradation and peak demand in one objective to show the trade-off between these three objectives. We do this to highlight the importance of a joint-decision making process where benefits must be shared to satisfy all involved stakeholders.

The framework of MOTEEO for case study 1, a H-MG is presented in Figure 1. Different stakeholders have business relationships (dashed link) with various participants of a smart grid (i.e. the EV owner, EV-O, owns the EVs and pays the DSO, who is in charge of the distribution system for the use of the grid, the policy maker P-M enforces environmental targets etc.). The components of the smart grid are modelled and these models are integrated within MOTEEO. In particular, EVs communicate their charging requirements, arrival and next departure times; these set the constraint of the optimisation. Within MOTEEO, a range of services/objectives are modelled according the necessities of the involved stakeholders. The decision variables that optimize the objectives are the EV charging scheduling. MOO is applied to provide the full range of available solutions. The stakeholders then participate in MCDM and the EV charging/discharging scheduling are decided. 


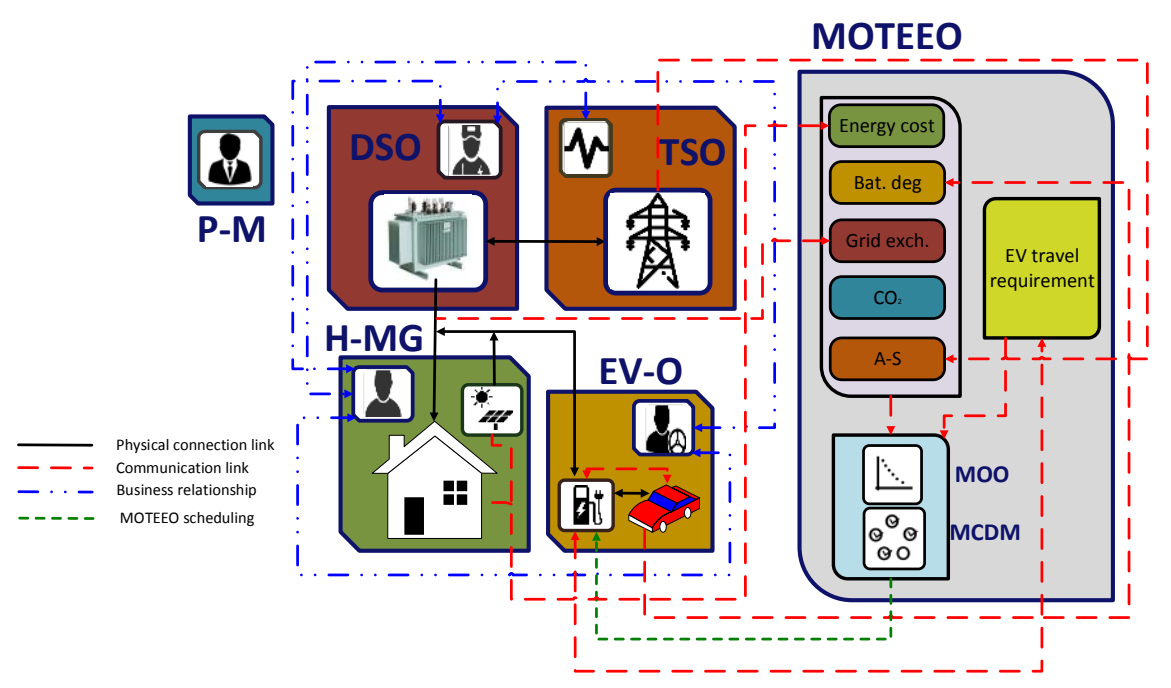

Figure 1 MOTEEO framework for single $H-M G$

The decentralised MOTEEO proposed in this paper is then applied to multiple EVs and dwellings in a real-distribution network as depicted in Figure 2. The business relationship links have not been depicted to simplify the image. Each EV applies MOTEEO, considering the objectives of the aforementioned stakeholders and the overall benefits are quantified. This case study is useful for the stakeholders at a higher level, i.e. DSO and policy maker, who can then quantify the prospective benefits at a higher scale than the single H-MG. These benefits are the reduction in the overall grid-peak demand and total $\mathrm{CO}_{2}$ emissions. Finally, in case study 3 , a utility function combining energy cost, battery degradation and grid net exchange is defined and optimised. This approach highlights the trade-off between the objectives and establishes the necessity of collaborative decision making. Stationary storage is also a promising technology which is extensively exploited in smart grids (see [16]). However, the aim of the current work is to highlight the opportunities brought by EVs. It is worth pointing out that the proposed MOTEEO framework is a consensus-based approach where EV users authorise the use of their EV batteries for energy services for a specific period and within certain energy levels; the algorithm ensures that the energy required by the EV user for the next trip is made available at the next departure. 


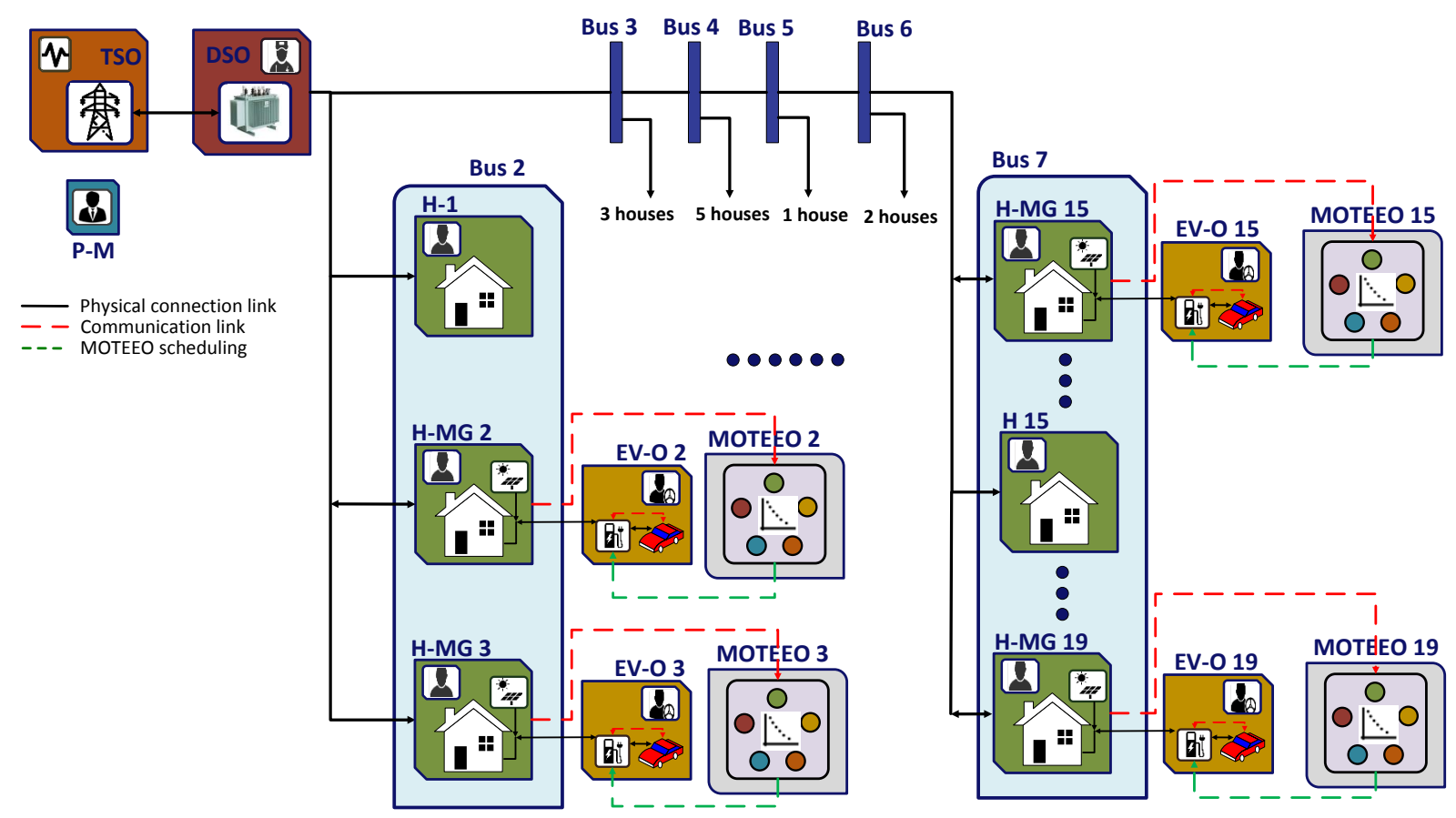

Figure 2 MOTEEO framework for a distribution network

\section{Assumptions for the mathematical model}

For the purpose of this research, a number of assumptions have been made while defining the mathematical mode. These apply to all the cases and scenarios.

- EVs have the same driving patterns as conventional internal combustion engine (ICE) vehicles.

- EV driving requirements are taken as constraints, and plug-in and plug-off times are approximated to the nearest quarter of an hour.

- The real-time electricity price provided to the consumer follows the same behaviour of the wholesale market price with distribution and transmission charges. This is not altered during EV charging.

- EV batteries reach end of EV life when their state of health drops to $80 \%$.

- Upon arrival at home, the SOC of the EV battery, departure time for the next trip and the required energy (distance to drive) are known.

- The daily dwelling electricity demand and PV generation profiles are known. It is assumed that prediction techniques can provide such information to the deterministic optimisation performed in this study.

- The utility company or an aggregator is responsible for providing electricity supply to the final customers, and also provides real-time pricing.

- An aggregator is responsible for the procurement of sufficient assets to meet the minimum requirement of EVs for frequency regulation. It is assumed that the aggregator is the DSO, so the revenue stream is directly passed from the DSO to the frequency regulation service providers. 
- Under smart charging and bidirectional charging, EV chargers can regulate the output power continuously.

- Houses are symmetrically distributed across the three phases of a $400 \mathrm{~V}$ feeder, therefore we analyse one phase.

- All charging events follow a constant current profile. Although real-life charging profiles also include constant-Voltage charging, this simplification does not diminish the quality of the modelled results as during constant-Voltage charging less energy is exchanged compared to constant-current charging.

- The impact of charging on battery degradation is equal to that of discharging.

- Calendar degradation does not influence the optimisation process and therefore is not quantified as a direct impact of the charging/discharging scheduling.

The above assumptions are aligned with the current market structures and state of the art; in fact, shortterm forecasting techniques achieve reasonable accuracy [24], hence demand and local generation profiles can be known day-ahead. There are examples of utility companies providing V2G services, where the EV user specifies the departure time and the required level of charge [25]. Companies with a portfolio of distributed energy providers are being developed with Nuvve being one of the major players [26]; they aggregate EV fleets to provide energy services and remunerate the EV owners. A DSO in the UK [27], is involved in major V $2 \mathrm{G}$ projects with the aim of reducing grid reinforcement costs. This highlights the interest of the system operators procuring V2G services by managing EV fleets. Calendar degradation is caused by high ambient temperature and SOC [28], with the former being unaffected by optimal charging scheduling. Moreover, the impact of SOC is quantified in the model developed in this work.

\section{Analytical formulation}

The stakeholders introduced in Section 2 pursue their objectives which can be economic, technical/operational and environmental. Figure 3 presents the flowchart for the proposed MOTEEO framework. In the present work, three different case studies, representing different scales and operating conditions are implemented. The four objectives, and one scenario introduced in Section 2, are mathematically formulated from Sections 3.1 to 3.5. The EV energy constraints, travelling requirements and limitations of the charging equipment are modelled in Section 3.6 and the augmented nondominated $\varepsilon$-constraint method (ANEC) is applied along with the Analytical hierarchy process (AHP) to quantify multiple optimal EV charging scheduling, in Sections 3.7 and 3.8 respectively. 


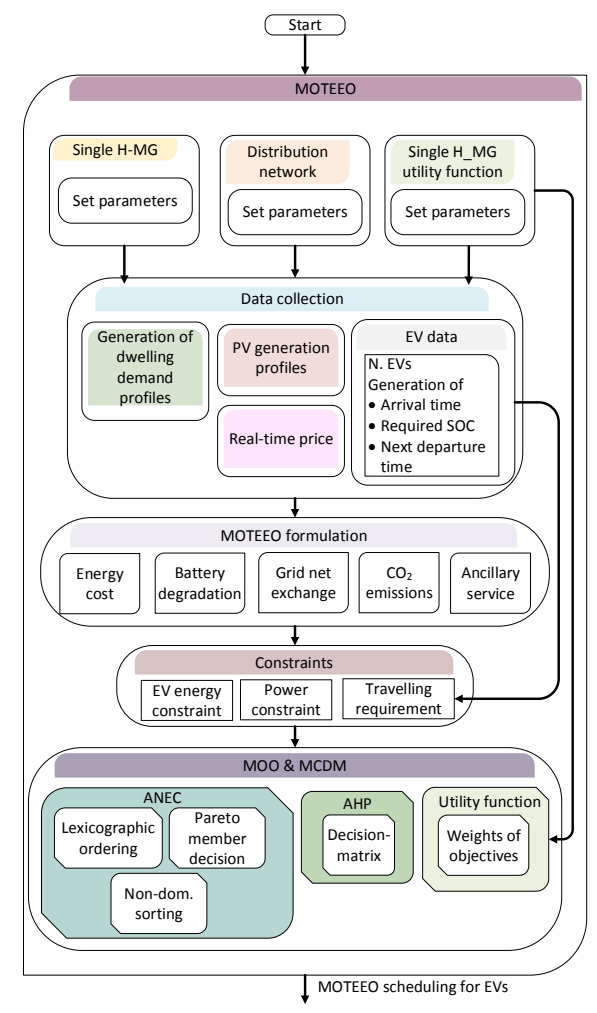

Figure 3 Flowchart of the proposed MOTEEO framework

\subsection{Energy cost minimisation}

From the point of view of the end electricity user, the operational cost represents a fundamental objective that has to be minimised in order to receive a return from the assets. Investments in energy efficiency and RES are made with the main aim of minimizing operational costs. For this study, a function representing the energy cost of the H-MG, $\mathbb{C}^{e}$, is expressed by Equation 1.

$$
\underset{P_{t}^{E V+}, P_{t}^{E V-}}{\operatorname{argmin}} \mathbb{C}^{e}=\sum_{t=1}^{N^{s}}\left[\left(P_{t}^{d}-P_{t}^{P V}+P_{t}^{E V+}-P_{t}^{E V-}\right) \Delta t \pi_{t}\right]
$$

Where $\pi_{t}$ is the price signal, $P_{t}^{d}$ is the electricity demand at time t, $P_{t}^{P V}$ is the PV generation at time t, $P_{t}^{E V+}$ is the power charged to the EV at time $\mathrm{t}$ and $P_{t}^{E V-}$ is the power discharged from the EV at time t. $\Delta t$ takes into account the energy exchanged in the time-step and $T^{s}$ is the total number of time steps considered in the scheduling. Here the decision variables are $P_{t}^{E V+}$ and $P_{t}^{E V-}$ : by iteratively manipulating their values, a minimum of the cost function for each time step can be reached.

\subsection{Battery degradation minimisation}

In this work, only cycling degradation is considered in the optimisation algorithm, as this is directly related to smart and bidirectional charging. Battery degradation $\operatorname{cost} C^{d}$ caused by a charging schedule is defined as: 


$$
\underset{P_{t}^{E V+}, P_{t}^{E V-}}{\operatorname{argmin}} \mathbb{C}^{d}=\sum_{t=1}^{N^{s}} \mathbb{C}_{t}^{d}\left(P_{t}^{E V+}, P_{t}^{E V-}\right)
$$

Where $\mathbb{C}_{t}^{d}=\frac{C^{B}}{E_{t}^{L}}$ is the battery degradation cost related to a charging/discharging event at time t, with $C^{B}$ being the investment cost of the battery in $£ / \mathrm{kWh}$ and $E_{t}^{L}$ the prospective lifetime energy throughput under certain charging condition $P_{t}^{E V+}, P_{t}^{E V-}$. It should be pointed out that the future cost of EV batteries is expected to decline and a range of values are forecasted. We have considered an average battery cost that is compatible with the future trends (see [29], [30], [31] and [32]). Furthermore, although new battery technologies are being developed for EV applications, the Global EV outlook 2018 of the International Energy Agency [1] expects that these will not reach commercialisation before 2030, and that lithium-ion batteries will represent the majority and continue to be used even after 2030 . From experiments performed on a range of Lithium-ion batteries, from cylindrical to automotive cells, cycled at a certain temperature, at a different charging/discharging rate and depth of discharge (DOD), $E_{t}^{L}$ can be defined, in accordance with [33], as:

$$
E_{t}^{L}=2 \times n^{E O L} \overline{E^{E V}} D O D
$$

Where $n^{E O L}$ is the number of full cycles before the battery reaches the End of life (EOL), $\overline{E^{E V}}$ is the maximum energy of the EV battery, DOD is the depth of discharge adopted in the tests (90\%), and a cycle is defined as a charging-discharging sequence (hence the 2 is employed in equation 3 ). It is observed from the empirical data [34] that capacity fading due to cycling degradation happens linearly with respect to the energy exchanged with the EV battery at each time step. EOL is generally associated with a battery degradation of $20 \%$, hence, $n^{E O L}$ can be defined as in Equation 4.

$$
n^{E O L}=\frac{0.2}{\alpha^{c}}
$$

Where $\alpha^{c}$ is the cycling degradation coefficient, which is influenced by several stress factors. In this paper, we model the battery degradation coefficient by considering three stress factors, namely battery temperature, charging rate and average SOC, in accordance with previous works [28], [35]. We propose an improved model based on [36] and adapted from cycling tests performed on commercial automotive Li-ion cells. In fact, in our improved model we fit the cycling results of automotive batteries and include the impact of average $S O C$, both not considered in [36]. In this study, we assume battery temperature to be the same as the average daily ambient temperature and is treated as an external condition that influences the optimisation but that cannot be handled to minimize its impact. Furthermore, the impact of charging and discharging rates are considered to be equal.

Equation 5 expresses the proposed battery degradation model

$$
\begin{aligned}
& \alpha^{c}\left(T^{B}, P_{t}^{E V+}, P_{t}^{E V-}\right)=\left[\beta_{1}\left(T^{B}\right)^{3}+\beta_{2}\left(T^{B}\right)^{2}+\beta_{3} T^{B}+\beta_{4}\right] \times\left[\beta_{5} C R+\beta_{6}\right] \times\left[\beta_{7}\langle S O C\rangle+\right. \\
& \left.\beta_{8}\right]
\end{aligned}
$$


where $\alpha^{c}$ is the capacity degradation coefficient due to cycling degradation, $T^{B}$ is the battery temperature, $C R=\frac{P_{t}^{E V+}}{\bar{E}^{E V}}$ is the charging/discharging rate, $\overline{E^{E V}}$ is the EV battery capacity, $\langle S O C\rangle$ is the average SOC of the battery during battery utilisation and $\beta_{1 \rightarrow 8}$ are fitting parameters. In equation 5 , the last multiplicative term considers the effect of EV charging at different average SOCs. In the simulated period, $\overline{S O C}$ depends on the defined decision variables based on equation 6 .

$$
\langle S O C\rangle=\frac{\operatorname{SOC}^{a}+\frac{\sum_{t=1}^{N}\left(\eta P_{t}^{E V+}-\frac{P_{t}^{E V-}}{\eta}\right) \Delta t}{\overline{E^{E V}}}}{T^{a}}
$$

where $S O C^{a}$ is the SOC of the EV upon arrival, $\Delta t$ is a time duration of a simulation step and $N^{a}$ is the total number of steps available from arrival until the next departure. By quantifying the battery degradation cost caused by a certain charging intervention, this can be compared against the associated benefit. Accordingly, the algorithm can choose the values of the decision variables, complying with the imposed constraints, to minimize degradation.

\subsection{Grid net exchange minimisation}

Storage solutions can minimize the time mismatch between RES generation and electricity demand, by charging in periods of RES excess and discharging in periods of high demand. In this way, the net power exchange profile with the grid is flattened which allows an optimised generation dispatch and stable grid operation. It is therefore in the DSOs' interest to allow energy storage implementation, both stationary and mobile (EV). The aim of the optimisation is to minimize the variation of the net power exchange with the grid. The objective function representing the grid net exchange $\mathbb{P}^{G}$, can be depicted as in Equation 7.

$$
\underset{P_{t}^{E V+}, P_{t}^{E V-}}{\operatorname{argmin}} \mathbb{P}^{G}=\sqrt{\sum_{t=1}^{N^{s}}\left(P_{t}^{d}-P_{t}^{P V}+P_{t}^{E V+}-P_{t}^{E V-}\right)^{2}}
$$

where $P_{t}^{d}, P_{t}^{P V}, P_{t}^{E V+}$ and $P_{t}^{E V-}$ assume the same meaning as in Equation 1. This objective function is calculated as the variation of the net power exchanged with the grid.

\section{4. $\mathrm{CO}_{2}$ emission minimisation}

EVs are seen as a major solution to reduce global $\mathrm{CO}_{2}$ emissions from the transportation sector. However, the environmental benefits of EVs depend on the carbon intensity of the national/local energy mix. This is because the energy mix that is used to charge the storage, and hence the $\mathrm{CO}_{2}$ emitted for energy provision, changes during the day, week and season. Therefore, there are periods of low specific $\mathrm{kgCO}_{2} / \mathrm{kWh}$ (off-peak), as opposed to periods with high specific $\mathrm{kgCO}_{2} / \mathrm{kWh}$ (peak). In this paper, the emissions avoided by ICE substitution are not considered.

Therefore, the objective function that aims to maximise environmental benefits can be defined in equation 8: 


$$
\underset{P_{t}^{E V+}, P_{t}^{E V-}}{\operatorname{argmin}} \mathbb{E}^{C O 2}=\sum_{t=1}^{N^{s}}\left[\frac{\left(P_{t}^{d}-P_{t}^{P V}+P_{t}^{E V+}-P_{t}^{E V-}\right)+\left(\sqrt{\left(P_{t}^{d}-P_{t}^{P V}+P_{t}^{E V+}-P_{t}^{E V-}\right)^{2}}\right)}{2} \Delta t e_{t}^{k W h}\right]
$$

where $e_{t}^{k W h}$ is the time series of the specific $\mathrm{CO}_{2}$ emission for each $\mathrm{kWh}$ absorbed from the grid. At present, these mechanisms are not adopted in the electricity industry, but with the increasing concern on greenhouse gas emissions, this method represents a suitable approach for the future.

\subsection{Revenues from ancillary service provision}

Ancillary services such as dynamic frequency regulation, require the regulation of the output of the generation/demand asset according to the frequency deviation from the nominal value of $50 \mathrm{~Hz}$. This is carried out with the droop control approach, which determines the regulation power provided in correspondence of a certain frequency deviation. When providing this service, EVs will have to follow the regulation signal defined Equation 9-11, subject to the same imposed constraints as for the other objectives:

$$
\begin{array}{ll}
\operatorname{reg}^{t}=-k^{d} f & \text { if } \underline{f} \leq f \leq \bar{f} \\
r e g^{t}=-\overline{P^{E V}} & \text { if } f \leq \underline{f} \\
r e g^{t}=\overline{P^{E V}} & f \geq \bar{f}
\end{array}
$$

Where $k^{d}=\frac{\overline{P^{E V}}}{(\bar{f}-\underline{f})}$ is the droop coefficient of the frequency controller, $f$ is the electrical frequency, $\bar{f}$ and $f$ are the upper and lower frequency limits of the droop-controller. We assume the EV provides Firm Frequency Regulation (FFR) over night from 11 p.m. to 7 a.m., which means in that period the $\mathrm{EV}$ is not available for other services. It is implied that availability for FFR should be compatible with the driving pattern of the EVs. According to [37], frequency regulation does not lead to net change in battery charge. Therefore, we assume that the SOC of an EV at the end of the FFR provision window is the same as the SOC at the beginning of that window. However, participation to FFR schemes implies battery utilisation, which leads to degradation. On the other hand, this service provides a remuneration. The interaction between this objective and the others will therefore be modelled based on the principles outlined as follows:

- The UK National Grid procures FFR for 8 hours on a daily basis. Providers can decide to deliver this service or not; to this end this decision is binary.

- The provision period is fixed and cannot be optimally distributed throughout the day according to a mathematical computation.

As a result of the above assumptions, the connection between Objective 1, 2, 3 and this scenario is brought to a binary decision variable $\left(F F R \in[0,1]_{\mathbb{N}}\right)$ which governs the FFR provision. Hence, we analyse two scenarios, with and without ancillary service provision. 


\subsection{Constraints of the optimisation - EV model}

The constraints for the various objectives presented so far that define the boundaries of the feasible region are presented. These are defined based on technical restrictions, usage behaviour as well as practical approach. The aim of the optimisation is to define power exchange profiles of EVs for different objectives, subject to constraints. Equations 12 to 15 link the power exchange of an EV with the energy stored.

$$
\begin{array}{ll}
E_{t}^{E V}=E^{E V, a} & \text { if } t=t^{a} \\
E_{t}^{E V}=E_{t-1}^{E V} & \text { if } A_{t}^{E V}=0 \\
E_{t}^{E V}=E_{t-1}^{E V}-E^{t r i p} & \text { if } t=t^{d}+1 \\
E_{t}^{E V}=E_{t-1}^{E V}+\left(\eta P_{t}^{E V+}-\frac{P_{t}^{E V-}}{\eta}\right) \Delta t & \text { if } A_{t}^{E V}=1
\end{array}
$$

In Equation 12, upon arrival of the EV, the energy stored in the battery is measured. In Equation 13, if the $\mathrm{EV}$ is not available $\left(A_{t}^{E V} \in[0,1]_{\mathbb{N}}\right.$ is a Boolean variable indicating the availability of the EV), then charging events cannot be initiated; hence, the energy state of the EV is unaltered ( $E_{t-1}^{E V}$ is the energy stored in the EV at the previous time step). Equation 14 takes into consideration the transportation constraint; in fact, at the departure time, the energy required for the next trip is deducted from the available capacity. If the EV is available, then in Equation 15 the energy stored is modified by adding the energy charged and deducting the energy discharged by taking into consideration the efficiency of the EV charger $\eta$.

The physical constraints in terms of storage size and power ratings as well as EV travelling requirement are presented from Equation 16 to 19.

$$
\begin{array}{ll}
0 \leq P_{t}^{E V+}, P_{t}^{E V-} \leq \overline{P^{E V}} & \forall \mathrm{t} \\
\frac{E^{E V}}{E_{t}^{E V}} \leq \overline{E^{E V}} & \forall \mathrm{t} \\
E_{t}^{E V} \geq E^{t r i p}+\underline{E^{E V}} & \text { if } t=t_{\text {dep }, n} \\
P_{t}^{E V+} \times P_{t}^{E V-}=0 & \forall \mathrm{t}
\end{array}
$$

Equations 16 and 17 limit the power exchanged by the EV and the energy stored within the respective bounds. Here, a minimum limit of EV capacity of $\underline{E^{E V}}=0.2 \overline{E^{E V}}$ has been set provide for unforeseen journeys. Equation 18 ensures that the energy stored in the EV meets the need of the user for the next trip. Finally, Equation 19 ensure that charging and discharging do not happen at the same time.

\subsection{Multi-objective optimisation with ANEC}

Some definitions are presented hereby to facilitate the formulation of the MOO problem [38].

Definition: Given a MOO problem expressed by Equation 20: 


$$
\min \boldsymbol{F}(\boldsymbol{x}): \Gamma \rightarrow \mathbb{R}^{k}=\left\{\begin{array}{c}
f_{1}(\boldsymbol{x}): \Gamma \rightarrow \mathbb{R} \\
\ldots \\
f_{k}(\boldsymbol{x}): \Gamma \rightarrow \mathbb{R}
\end{array} \quad k \geq 2\right.
$$

where $\Gamma \subseteq \mathbb{R}^{n}$ is the feasible region, defined by the imposed constraints and $f_{1}, \ldots, f_{k}$ is the set of objectives

- a solution $\boldsymbol{x}^{\prime}$ is said to Pareto dominate another solution $\boldsymbol{x}$ and is indicated as $\boldsymbol{x}<\boldsymbol{x}^{\prime}$ if

- $f_{i}\left(\boldsymbol{x}^{\prime}\right) \leq f_{i}(\boldsymbol{x}) \quad \forall i=1, \ldots, k$

- $f_{j}\left(\boldsymbol{x}^{\prime}\right)<f_{j}(\boldsymbol{x})$ for at least one $j=1, \ldots, k$

- a solution $\boldsymbol{x}^{\prime}$ is Pareto optimal/efficient/non-dominated if there is no other solution that dominates it. The Pareto front is the set of all the Pareto optimal solutions.

- In other words, Pareto optimal solutions are those that cannot be improved along one objectives without deteriorating the performance along another objective. The aim is to produce all the Pareto optimal solutions in order to enable decision-making.

Among strategies aimed at obtaining the full Pareto front, the augmented $\varepsilon$-constraint method is widely implemented [39]-[43], for fast and reliable MOO. With this approach, one objective is optimised while the others are converted into constraints. By varying the strictness of such constraints, a subset of the Pareto front can be obtained. In this algorithm, Lexicographic ordering is applied to define the range of the objective values, from their maxima to the minima. Under Lexicographic ordering, the objectives are given priorities and are sequentially optimised; the values from the optimisations at higher priorities are used as constraints for the optimisations at lower levels. We subsequently apply non-dominated sorting to ensure that all the solutions are Pareto efficient. The following pseudo-code outlines (ANEC).

Table 2 Pseudo-code for ANEC






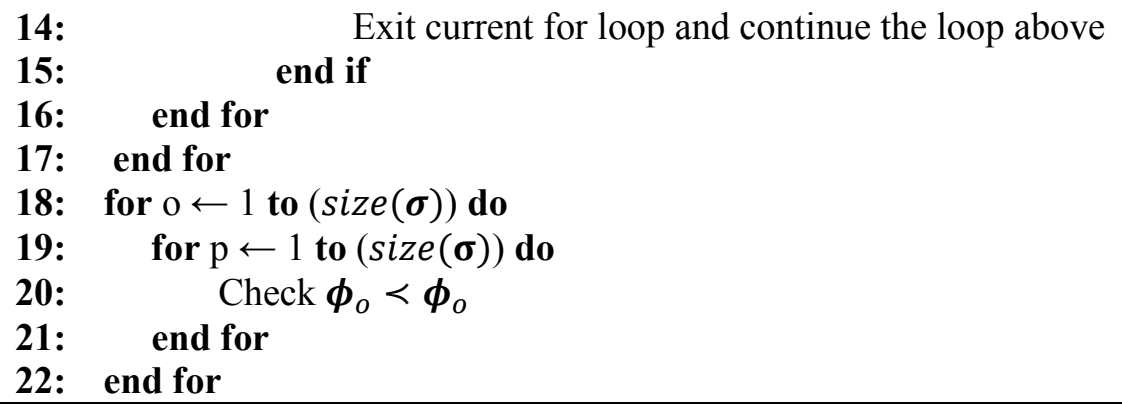

Where $f^{k}$ is the prioritised objective during Lexicographic ordering, $\boldsymbol{q}$ is the index vector for the nested for loops, $\boldsymbol{\varepsilon}$ is a vector representing the constraints for the objective functions that are not minimised. There will be as many nested for loops as $n^{f o r}=m-1 . S=\left[s_{1}, \ldots, s_{m-1}\right]$ are the slack-variables adopted for the augmented- $\varepsilon$ constraint and $\gamma$ is an arbitrary constant value . As the Pareto optimal solutions are progressively calculated, the values of $\boldsymbol{\varepsilon}$ vary from the maxima of the single objective functions to the minima. $\boldsymbol{\phi}_{o}$ and $\boldsymbol{\phi}_{p}$ are solutions of the $\varepsilon$-constraint method. It should be noted that $n^{\max }$ is the number of divisions of the range of each objective values. As the $\varepsilon$ constraint for one objective is varied within the for loop, the constraints of the other objectives are kept constant. In the for loops at higher levels, the $\varepsilon$ constraints of the other objectives are varied. It is evident that a number of computations will be infeasible; this is because as the objectives are conflicting and the objective values are constrained from their maxima to the minima, two conflicting objectives cannot simultaneously reach their minimum values. To avoid unnecessary computations, once an infeasible computation is found, the current for loop is ended and the loop at the higher level is continued.

\subsection{Multi-criteria-decision-making with analytical hierarchy process}

Once the full Pareto front is obtained, a decision needs to be taken to choose the preferred solution. If no preference is shown, the Pareto front represents the set of solutions that are equally optimal and therefore equivalent. MCDM techniques can help on choosing one solution from the Pareto front. In this study AHP [44] is employed.

AHP evaluates the performance of $n$ alternative solutions along a set of $m$ objectives. The decision maker prioritizes the different objectives with a relative comparison matrix $\boldsymbol{A}_{m \times m}$. The priority of each objective is quantified with relations $A_{i j} . A_{i i}=1, \forall i$ as an objective has the same priority as itself. The relative comparison of two different objectives is outlined as follows: if $i$ is more important than $j$, then:

$$
\left\{\begin{array}{l}
A_{i j}=k, \\
A_{j i}=\frac{1}{k}
\end{array}\right.
$$

Where $k \in[1,9]$ determines the relative priority of $i$ compared to $j ; A_{i j}=1$ indicates that $i$ and $j$ have the same importance while $A_{i j}=1$ indicates that $i$ is extremely important compared to $j$. Then, a vector of weights $\boldsymbol{B}_{m x 1}$ is built and ultimately, a final score is assigned to each solution based on their 
performance along each objective. The solution having the highest score shall be chosen as the preferred solution. It should be pointed out that, since in this paper we consider three decision makers, 3 weight vectors will be individually considered, i.e. $\left[\boldsymbol{B}^{1} \boldsymbol{B}^{2} \boldsymbol{B}^{3}\right]$. An example of common decision making processes in a group of stakeholders with diverse and conflicting objectives can be found in [45], where a method to combine the priorities of the different groups of decision makers in one matrix is proposed. However, in the present work, each group of decision makers is modelled separately, to highlight the benefits/drawbacks of different prioritisations and, as will be discussed in Section 4.3, a profit sharing approach to mitigate conflicts is proposed. A consistency check is also performed for each pairwise decision matrix to ensure reliability of the decision making process; a detailed explanation is provided in [44].The priorities of a group of stakeholders are modelled assuming that there is homogeneity in each group, namely end-electricity-users, EV users and DSO. Certainly, there will be differences in the priorities of each individual, which could be captured with a survey. However, in depth modelling of detailed user preference need to cover a dedicated investigation, which is beyond the scope of the paper.

\section{Results and discussion}

The proposed MOTEEO framework is initially applied at a household level to demonstrate the effectiveness of proposed method to model and maximize the interests of the five stakeholders. Subsequently, the strategy is applied to a typical distribution network with realistic penetration level of PV systems and EVs. Three EV charging strategies are adopted: uncontrolled, smart and bidirectional charging. The three DM who are involved in the decision-making process are the end electricity user, the EV owner and the DSO. Finally, an alternative utility function based MOTEEO is applied for a single-household to show the importance of collaborative decisions where benefits are shared.

\subsection{Case study setting}

As introduced in Figure 1, a single-dwelling comprising of a 4kW PV installation and one 30kWh EV is considered for case study 1 and case study 3 and the associated parameters are detailed in Table 3 .

Table 3 Setting for the case study 1

\begin{tabular}{ll}
\hline Parameters & \\
\hline Electricity demand & Detached single-house, single-phase \\
RES type & Roof-top photovoltaic \\
RES system rating & $4 \mathrm{~kW}$ \\
$\boldsymbol{C}^{\boldsymbol{B}}$ & $150 £ / \mathrm{kWh}$ \\
EV charger type & Type 2 conventional/Smart/Bidirectional/ single-phase \\
$\overline{\boldsymbol{P}^{\boldsymbol{E}}}$ & $3 \mathrm{~kW}$ \\
$\boldsymbol{\eta}$ & $90(90) \%$ \\
$\Delta \boldsymbol{t}$ & $15 \mathrm{~min}$ \\
Pricing strategy & Real-time pricing \\
Optimisation strategy & Day-ahead \\
$\boldsymbol{T}^{\boldsymbol{B}}$ & $18 \mathrm{C}$ \\
$\boldsymbol{t}^{\boldsymbol{a}}$ & $17: 00$
\end{tabular}




$\begin{array}{ll}\boldsymbol{t}^{\boldsymbol{d}} & 10: 15 \\ \boldsymbol{E}^{\boldsymbol{E V}} & 30 \mathrm{kWh}\end{array}$

The optimisation is performed one day-ahead, with a real-time price derived from the wholesale spot price by adding network charges and taxes [46]. From the modelling implemented in Section 3.5, two scenarios, with and without ancillary service provision, are simulated. Figure 4 depicts the evolution of the real-time price, and EV availability for case study loptimisation. It can be demonstrated that when $\mathrm{PV}$ generation is available, minimising grid net exchange corresponds to minimising $\mathrm{CO}_{2}$ emissions. A practical demonstration is provided in the appendix. Consequently, we minimize Objective 1 - Energy cost, Objective 2 - EV battery degradation and Objective 3 - Grid net exchange. The mathematical optimisation process for case study 1 is hereby detailed.

A) $\quad \zeta_{m \times m}^{l e x}=\operatorname{lexmin}_{x, f^{k}} F^{m} \quad \mathrm{k}=1,2,3$ and $\mathrm{m}=3$, where $f^{k}$ has the highest priority.

B) We define:

$\Psi_{i}=\max \left(\zeta_{i}^{\text {lex }}\right)$ Nadir point and $\psi_{i}=\min \left(\zeta_{i}^{\text {lex }}\right)$ for $\mathrm{i}=1,2,3$. Where $\zeta_{i}^{\text {lex }}$ are the results for objective $i$ from the Lexicographic ordering.

C) for $o=1, \ldots, n^{\max }+1$ and $p=1, \ldots, n^{\max }+1 \mathrm{We}$ minimise:

D) $\underset{P_{t}^{E V+}, P_{t}^{E V-}, s_{2}, s_{3}}{\operatorname{argmin}} \mathbb{C}^{e}=\sum_{t=1}^{N^{s}}\left[\left(P_{t}^{d}-P_{t}^{P V}+P_{t}^{E V+}-P_{t}^{E V-}\right) \Delta t \pi_{t}\right]-\gamma\left(s_{2}+s_{3}\right)$

E) Subject to (12) to (19) and

F) $\quad \mathbb{C}^{d}=\varepsilon_{2}+s_{2}$

G) $\quad \mathbb{P}^{G}=\varepsilon_{3}+s_{3}$

Where

H) $\quad \varepsilon_{2}=o \times \frac{\Psi_{2}-\psi_{2}}{n^{\max }}, \varepsilon_{3}=p \times \frac{\Psi_{3}-\psi_{3}}{n^{\max }}$ with $n^{\max }=6, s_{2}$ and $s_{3}$ are slack variables and $\gamma$ is an arbitrary constant

I) If FFR is provided, $A_{t}^{E V}=0$ from 23 to 7.

J) end for

For case study 2, we apply the setting for case study 1 to all the EVs involved, in compliance with the associated electricity demand profiles, $\mathrm{PV}$ generation and EV transportation requirements.

For case study 3 , we adopt the same setting outlined in Table 3 but with the implementation of a utility function; the mathematical process is as follows:

A) $\underset{P_{t}^{E V+}, P_{t}^{E V-}}{\operatorname{arggmin}} \lambda_{1} \mathbb{C}^{e}+\lambda_{2} \mathbb{C}^{d}+\lambda_{3} \mathbb{P}^{G}$

B) Subject to (12) to (19)

Where $\lambda_{1}=1, \lambda_{2}=C^{B}=150$ and $\lambda_{3}$ depends on the grid utilisation fee set by the DSO 
All the simulations have been carried out on a computer with an Intel Core i7-6500U CPU $2.5 \mathrm{GHz}$ processor and 16GB RAM Time resolution for the optimisation in all case studies is $15 \mathrm{~min}$. Sequential quadratic programming algorithm in Matlab2017a has been employed for the non-linear optimizations.

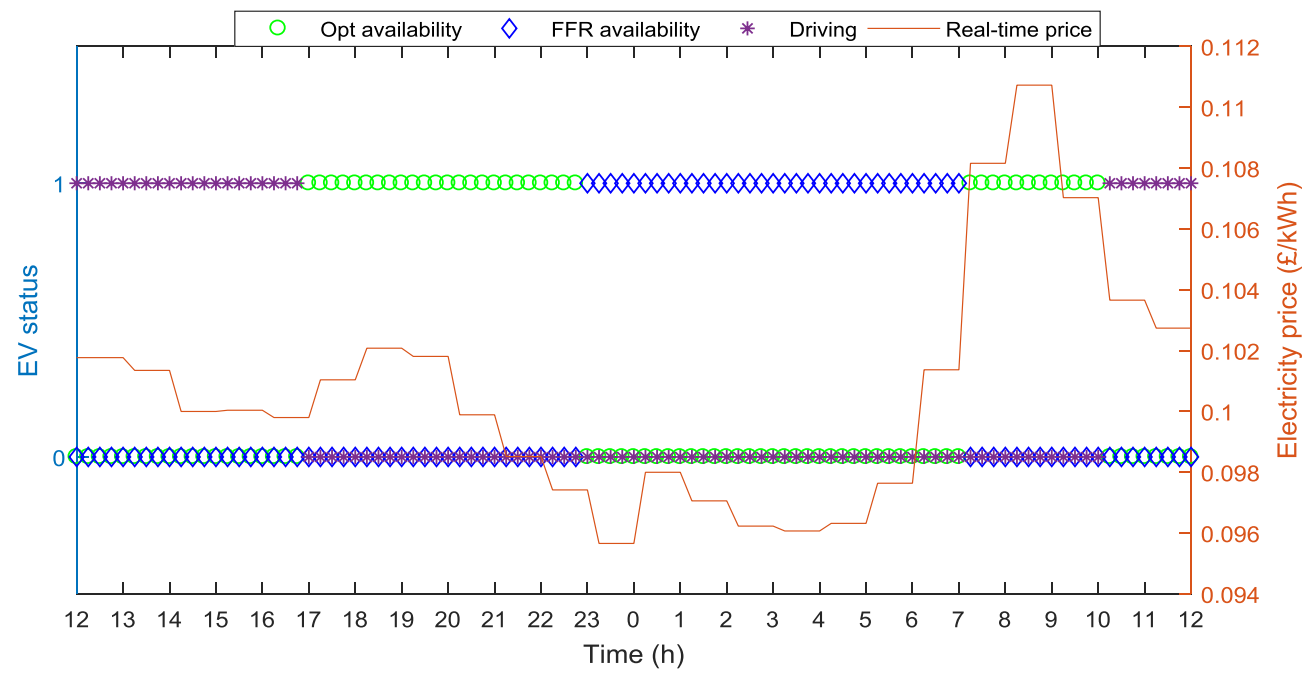

Figure 4 EV availability and real-time price

As depicted in Figure 2, a typical UK distribution network (DN) [47], comprising a $400 \mathrm{~V}$ feeder which provides electricity to 57 houses is considered for case study 2. One phase of the $400 \mathrm{~V}$ feeder is simulated assuming balanced three-phase load distribution therefore, 19 houses are individually simulated. To quantify the unbalance in a three-phase distribution system, the three phases should be individually simulated and the conclusions, which are rather network and location dependent, should be evaluated on a case-by-case basis [48]. Eight days from four seasons, considering both week-days and week-ends, have been investigated. Different PV generation profiles, depicting the seasonal variations, and different EV availability patterns have been considered [49]. All the electricity demand profiles have been generated from the Centre for Renewable Energy Systems Technology (CREST) model [50]. The configuration of the typical DN is based on the PV and EV penetrations levels predicted for 2040 [51]. The year 2040 represents a crucial landmark because of the ban of ICE vehicles announced by the UK government [52]. By considering the current penetration of domestic PV systems [53], [54] and using the prediction of the UK National Grid Future energy scenario [51], a penetration rate of $50 \%$ is projected. This implies that in one phase of the LV feeder, 10 houses will be equipped with a PV system. Since the UK average PV system size is $3.35 \mathrm{~kW}$, a normal distribution around a mean value of $3 \mathrm{~kW}$ is assumed. The PV generation profile is referred to a typical winter day (hence the ambient temperature) of the Midlands in the UK. An EV penetration rate of $50 \%$ is expected for 2040 [51], among those that have access to at least one car [55] hence, 10 EVs are simulated. Table 4 lists the parameters adopted to produce the EV transportation model and other key assumptions for case study 2 . 


\begin{tabular}{ll}
\hline Parameter & Value \\
\hline EV and PV penetration rate & $50 \%[51],[53],[54]$ \\
Average daily mileage & 9 miles [55], [56] \\
Average daily energy & $1.74 \mathrm{kWh}[55],[56]$ \\
consumption & \\
Arrival and departure times for trips randomly selected from National Time use Survey data \\
Average PV size & $3.35 \mathrm{kWp} \mathrm{[53]}$ \\
$\overline{E^{E V}} / \overline{P^{E V}}$ & $30 \mathrm{kWh} / 3 \mathrm{~kW}$ \\
EV charger type & Type 2 conventional/Smart/Bidirectional/ single-phase \\
Frequency regulation prices & From UK National Grid post-tender reports \\
\hline
\end{tabular}

\subsection{Results of case study 1 for single H-MG}

To demonstrate the effectiveness of MOTEEO, highlighting the conflict of the different objectives defined in section 3, we apply the proposed methodology to a single dwelling with one EV.

\section{Single objective optimisation algorithm}

Figure 5 shows EV charging scheduling for Objectives 1, 2 and 3 without FFR provision.

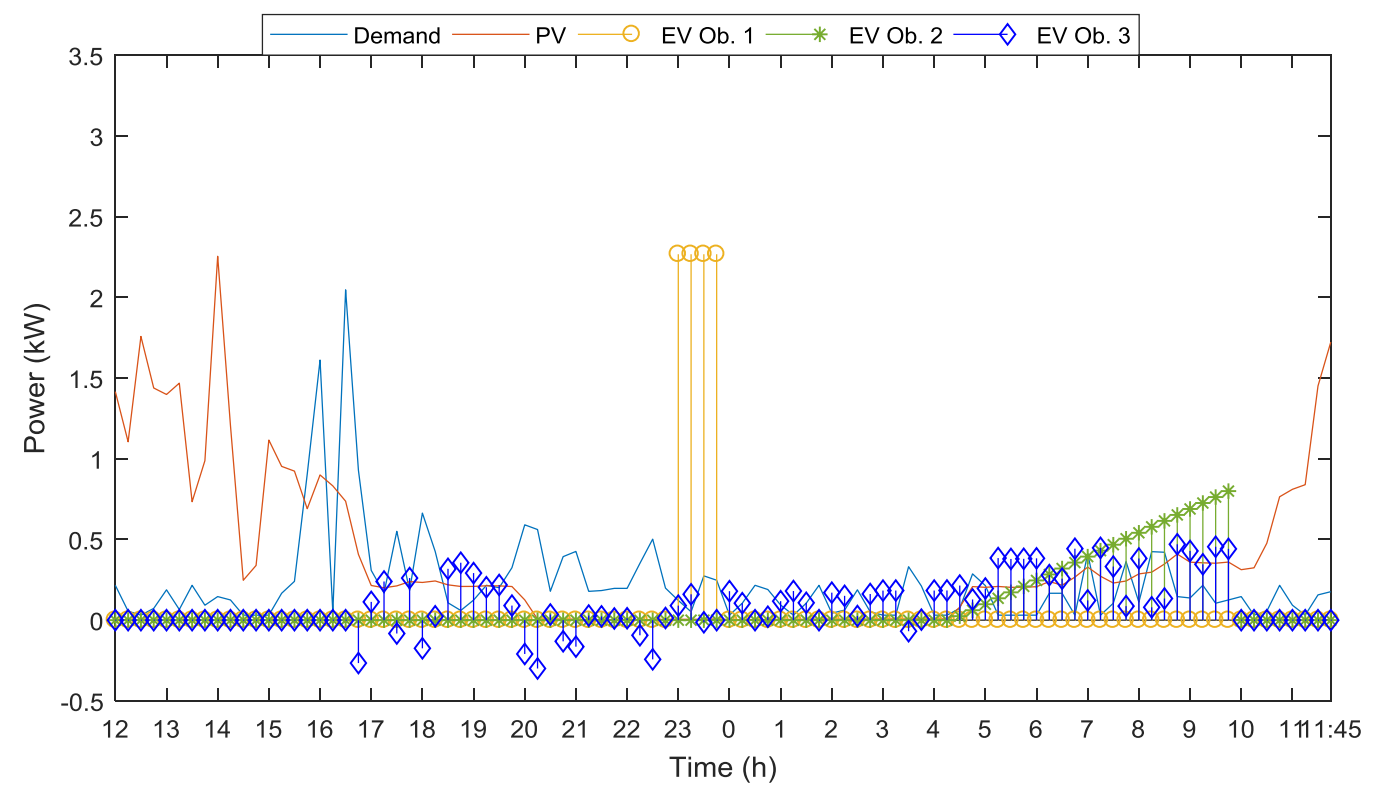

Figure 5 EV scheduling for single-objective optimisations without FFR

With reference to the cost signal in Figure 4, it can be seen that under Objective 1 the EV is charged at the minimum price available. Further price arbitrage is not carried out since having a round-trip efficiency of $81 \%$ the price difference is not sufficiently profitable. Furthermore, the transportation constraints are satisfied as the EV is charged before the next departure. When the EV is charged to minimize battery degradation under Objective 2, the charging happens only close to the next departure to minimize average SOC. In addition, the charging rate is gradually increased to minimize degradation. This is because from equation 5, the combination of high charging rate and high average SOC causes high degradation; charging the battery at a lower constant charging rate would have increased charging 
duration leading to a higher average SOC and therefore degradation. At the same time, the full charging rate $(3 \mathrm{~kW})$ is not employed as it would increase degradation; an optimum solution which underlines a balance between the charging rate and the average SOC (their product is considered in equation 5) is found. Under Objective 3, the EV is used to minimize the grid net exchange. As during the PV excess hours the EV is mostly absent, PV energy autonomy is not fully maximised. However, upon arrival, the EV exploits as much PV energy as possible and the peaks of electricity demands are also provided by discharging the EV. Here, the conflict between the different objectives are unveiled. In fact, the EV is charged with radically different scheduling under the three objectives and the scheduling according to one objective inevitably worsen the performance along the others.

Figure 6 depicts EV charging scheduling for Objectives 1, 2 and 3 with FFR provision. It should be noted that the EV does not initiate any charging event from 11 p.m. to 7 a.m., in accordance with the FFR commitment.

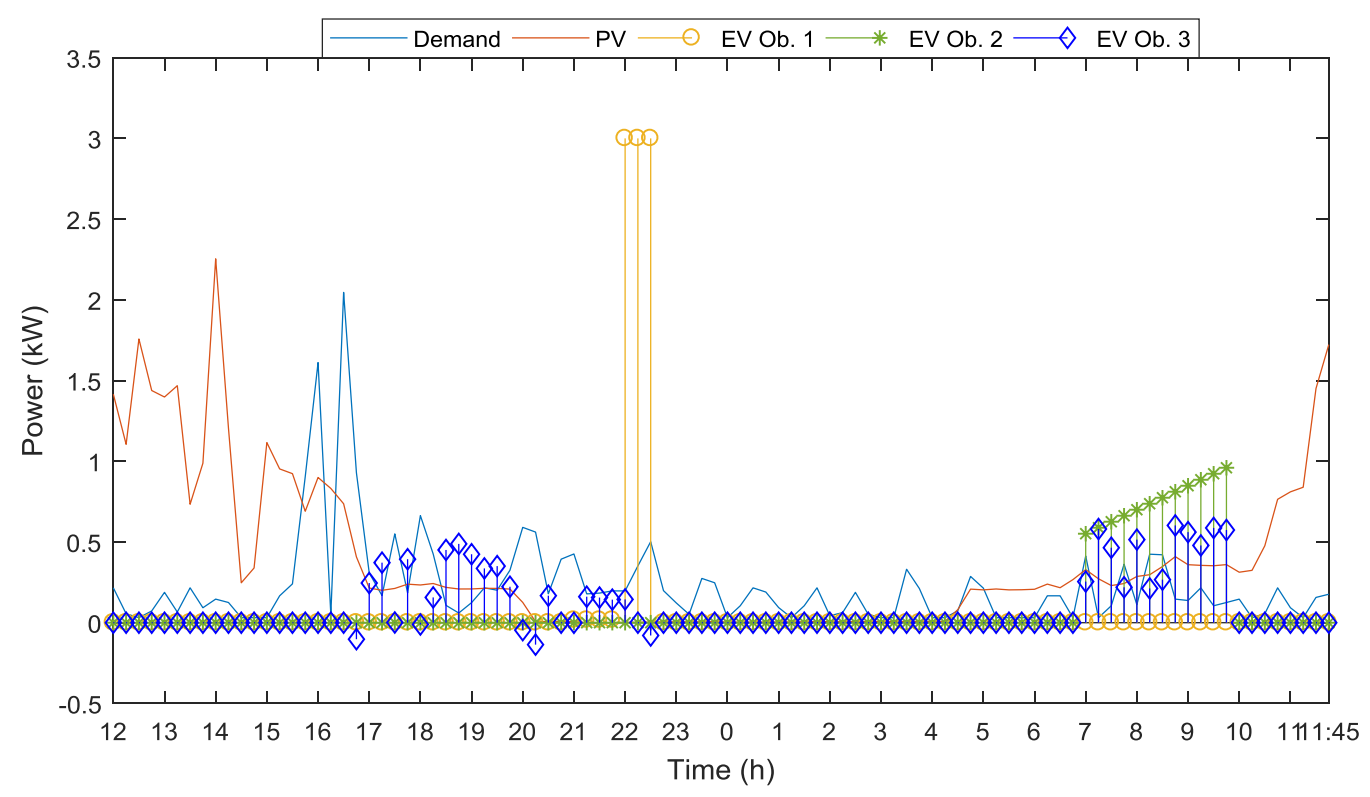

Figure 6 EV scheduling for single-objective optimisation with FFR

As shown in Figure 6, under Objective 1, the EV charging happens right before the FFR window starts (between 10-11 pm, which provides the lowest price in the available window). As for Objective 2, the charging is concentrated in the 7-10 period at a higher charging rate than Scenario i), which leads to higher degradation. Under all objectives, the EV is charged before the FFR window, which keeps the EV at a higher SOC, leading to a higher battery degradation compared to scenario i). In addition, the performance under Objective 3 is worse as there is less availability of the EV to service/meet the electricity demand. Table 5 presents the results of the three single optimizations with and without FFR provision. Throughout this paper, costs have been designated with negative sign while revenues have been attributed a positive sign. 
Table 5 Results of the single-objective optimisations for one day

\begin{tabular}{|c|c|c|c|c|}
\hline & \multicolumn{4}{|c|}{ Scenario i) (without FFR provision) } \\
\hline & $\begin{array}{l}\text { Energy } \\
\operatorname{cost}(£)\end{array}$ & $\begin{array}{l}\text { Battery } \\
\text { degradation }(\mathfrak{£})\end{array}$ & $\begin{array}{l}\text { Grid net } \\
\text { exchange }(\mathrm{kWh})\end{array}$ & $\begin{array}{l}\text { Emissions } \\
\left(\mathrm{kgCO}_{2}\right)\end{array}$ \\
\hline $\min O b j_{1}$ & 0.1617 & -0.0127 & 11.3 & 1.6394 \\
\hline $\min O b j_{2}$ & 0.1366 & -0.0118 & 9.9647 & 1.4559 \\
\hline $\min O b j_{3}$ & 0.1353 & -0.0170 & 9.8243 & 1.4197 \\
\hline & \multicolumn{4}{|c|}{ Scenario ii) (with FFR provision) } \\
\hline & $\begin{array}{l}\text { Energy } \\
\operatorname{cost}(£)\end{array}$ & $\begin{array}{l}\text { Battery } \\
\text { degradation }(\mathfrak{f})\end{array}$ & $\begin{array}{l}\text { Grid net } \\
\text { exchange }(\mathrm{kWh})\end{array}$ & $\begin{array}{l}\text { Emissions } \\
\left(\mathrm{kgCO}_{2}\right)\end{array}$ \\
\hline $\min O b j_{1}$ & 0.7947 & -0.1031 & 11.3 & 1.6891 \\
\hline $\min O b j_{2}$ & 0.7695 & -0.1020 & 10.4762 & 1.5469 \\
\hline $\min O b j_{3}$ & 0.7742 & -0.1034 & 10.2768 & 1.5521 \\
\hline
\end{tabular}

When FFR is provided, the energy cost is further reduced by the FFR profits (£0.637) and battery degradation increased (£0.0902), resulting in an overall profitable service. As for the fourth objective, the limitation of the available optimisation window due to FFR provision increases the $\mathrm{CO}_{2}$ emissions slightly. Once the conflict between the objectives have been highlighted, MOO and MCDM techniques are applied to find the optimal solutions for all the three objectives.

\section{MOTEEO optimisation algorithm}

Figure 7 and Figure 8 depict the Pareto fronts obtained from the ANEC method for scenarios i) and ii). The performance along the three objectives have been normalised to their maximum values expressed in Table 5 to allow comparative analysis. $n^{\max }$ was set to 6 , which leads to a maximum of 49 Pareto efficient solutions. However, as discussed in Section 3.7, due to the conflict among objectives, a number of computations were infeasible, and this led to 42 and 35 Pareto efficient solutions for scenarios i) and ii), respectively. Some of the solutions cumulate in certain points; this could be avoided by dividing the solutions space with higher resolution; however, this would increase the computational cost [defined as $O\left(\left(n^{\max }+1\right)^{2}\right]$. In this study, a rightful combination of both enough granularity of the Pareto front to informatively take decisions and computational cost has been achieved. 


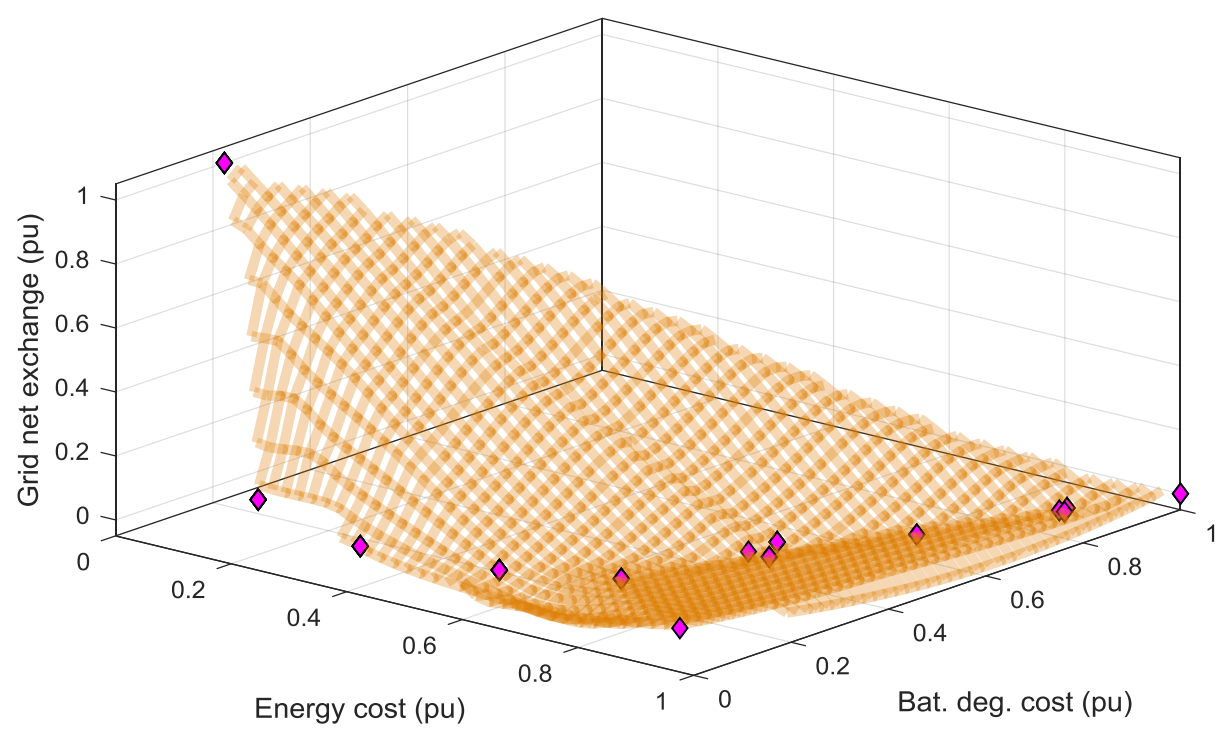

Figure 7 Pareto front with ANEC method without FFR

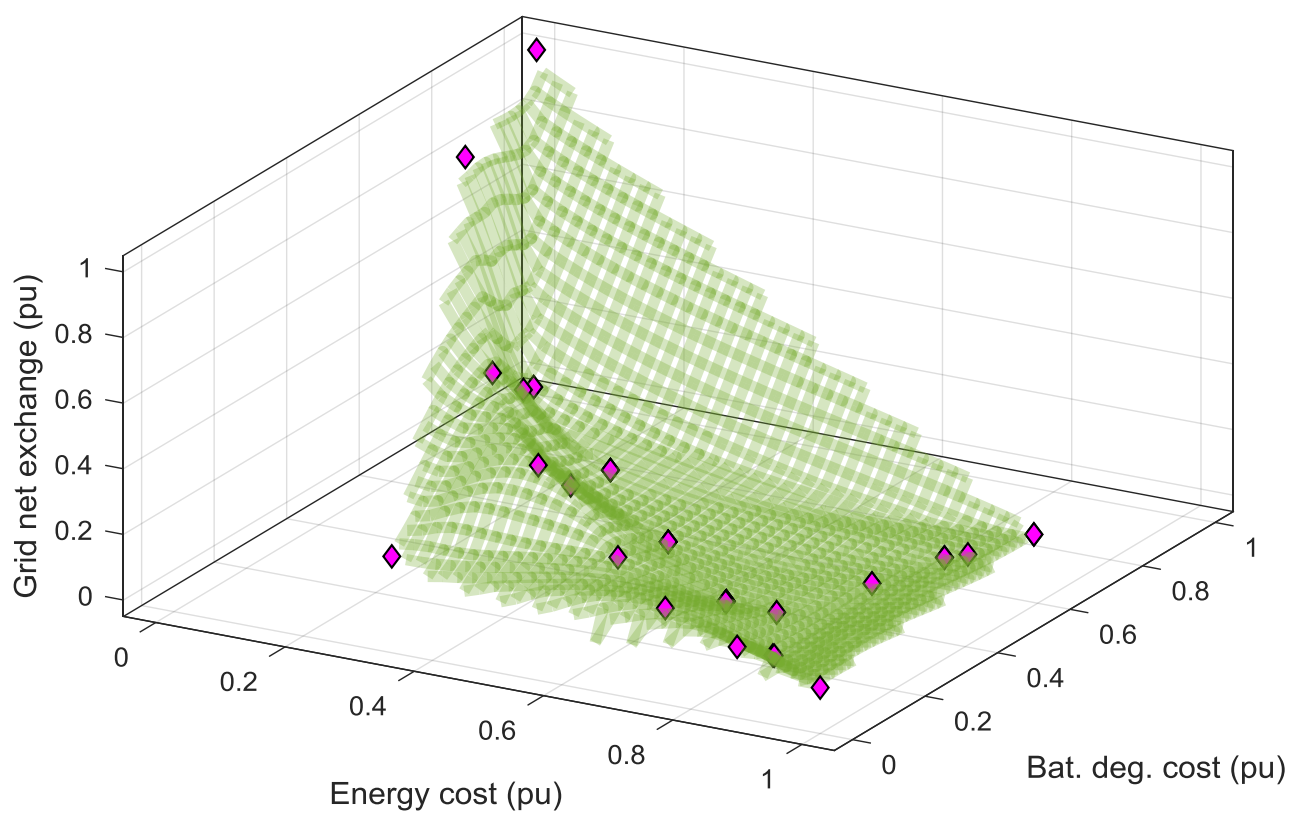

Figure 8 Pareto front with ANEC method and FFR

It can be observed in Figure 8 that in one area of the solutions space, minimising grid net exchange also leads to battery degradation minimisation. This is because when FFR is provided, in order to minimise energy cost, the algorithm schedules EV charging at $3 \mathrm{~kW}$ during the minimum price period available (see Figure 6), which increases both battery degradation and grid net exchange (see Table 5). When battery degradation is forced to be reduced by the $\varepsilon$-constraint, the charging scheduling tends to the behaviour of EV Ob. 2 (green stems) which is closer to the behaviour of EV Ob. 3 (blue stems), therefore reducing grid net exchange. However, when grid net exchange is forced to be reduced towards its 
minimum value, battery degradation is increased. AHP is applied to choose the optimal solutions among the Pareto members provided by the MOO, according to the different prioritisation of the stakeholders. Three stakeholders/decision makers (DM) holding different priorities are considered. The relative comparison matrices (following from Section 3.8) for the three DMs is shown in Table 6.

Table 6 Relative comparison matrix for different DMs

\begin{tabular}{|c|c|c|c|c|c|c|c|c|c|c|c|}
\hline & \multicolumn{3}{|c|}{$D M_{1}$} & \multicolumn{4}{|c|}{$D M_{2}$} & \multicolumn{4}{|c|}{$\mathrm{DM}_{3}$} \\
\hline$O b_{1}$ & $\begin{array}{c}O b_{1} \\
1\end{array}$ & $\begin{array}{c}O b_{2} \\
5\end{array}$ & $\begin{array}{c}\mathrm{Ob}_{3} \\
\text { 列 }\end{array}$ & $O b_{1}$ & $\begin{array}{c}O b_{1} \\
1\end{array}$ & $\begin{array}{c}O \mathrm{Ob}_{2} \\
\frac{1}{5}\end{array}$ & $\begin{array}{c}\mathrm{Ob}_{3} \\
2\end{array}$ & $O b_{1}$ & $\begin{array}{c}O b_{1} \\
1\end{array}$ & $\begin{array}{c}\mathrm{Ob}_{2} \\
\frac{1}{2}\end{array}$ & $\begin{array}{c}\mathrm{Ob}_{3} \\
\frac{1}{9}\end{array}$ \\
\hline$O b_{2}$ & $\frac{1}{5}$ & 1 & 2 & $O b_{2}$ & 5 & 1 & 9 & $O b_{2}$ & 2 & 1 & $\frac{1}{5}$ \\
\hline$O b_{3}$ & $\frac{1}{9}$ & $\frac{1}{2}$ & 1 & $O b_{3}$ & $\frac{1}{2}$ & $\frac{1}{9}$ & 1 & $O b_{3}$ & 9 & 5 & 1 \\
\hline
\end{tabular}

$D M_{1}$ is the end-electricity user who wants to minimize the energy cost. $D M_{2}$ Is the EV owner who wants optimize the exploitation of the EV battery and $D M_{3}$ is represented by the DSO or the policy maker who wants to optimize grid utilisation and minimize $\mathrm{CO}_{2}$ emissions. The consistency ratios found for the three pairwise decision matrices, related to the three DMs, are lower than 0.1 which verifies the consistency of the decisions It should be pointed out that multi-objective optimisation applied to EV charging scheduling has only recently gained interest in the research community. Hence, there is a lack of studies addressing the prioritisation adopted by the different stakeholders, especially the EV user, for the different objectives. Thus, the priorities in the three relative comparison matrices have been set based on suitable prioritisation rules and could be verified by surveying a heterogeneous sample of potential stakeholders.

The results from the decision making process are shown in Table 7. It can be seen that MOO with MCDM finds the overall best option while still favouring the DM's choice. This is because once the full Pareto set is available, there is more freedom on choosing the option that achieve the best performance along the objectives while complying with the inherent prioritisation of the stakeholder.

Table 7 Results of the MOTEEO method with the application of AHP for one day

\begin{tabular}{|c|c|c|c|c|}
\hline & \multicolumn{4}{|c|}{ Scenario $i$ ) (without FFR provision) } \\
\hline & $\begin{array}{l}\text { Energy } \\
\operatorname{cost}(£)\end{array}$ & $\begin{array}{l}\text { Battery } \\
\text { degradation }(\mathfrak{f})\end{array}$ & $\begin{array}{l}\text { Grid net } \\
\text { exchange }(\mathrm{kWh})\end{array}$ & $\begin{array}{l}\text { Emissions } \\
\left(\mathrm{kgCO}_{2}\right)\end{array}$ \\
\hline $\mathbf{D M}_{1}$ & 0.16 & -0.0127 & 11.3 & 1.64 \\
\hline $\mathbf{D M}_{2}$ & 0.15 & -0.0120 & 9.95 & 1.42 \\
\hline \multirow[t]{3}{*}{$\mathbf{D M}_{3}$} & 0.15 & -0.0120 & 9.95 & 1.42 \\
\hline & \multicolumn{4}{|c|}{ Scenario ii) (with FFR provision) } \\
\hline & $\begin{array}{l}\text { Energy } \\
\text { cost }(£)\end{array}$ & $\begin{array}{l}\text { Battery } \\
\text { degradation }(£)\end{array}$ & $\begin{array}{l}\text { Grid net } \\
\text { exchange (kWh) }\end{array}$ & $\begin{array}{l}\text { Emissions } \\
\left(\mathrm{kgCO}_{2}\right)\end{array}$ \\
\hline $\mathbf{D M}_{1}$ & 0.794 & -0.1022 & 10.54 & 1.49 \\
\hline $\mathbf{D M}_{2}$ & 0.769 & -0.1020 & 10.48 & 1.55 \\
\hline
\end{tabular}


From the results, it can be seen that the stakeholders would choose the solution that naturally fits with their priorities, sacrificing the performance along other objectives. Comparing Table 7 with Table 5, some differences are visible. Under scenario i), $\mathrm{DM}_{2}$ and $\mathrm{DM}_{3}$ agree on the same solution. When providing FFR, $\mathrm{DM}_{3}$ chose a solution that caused lower battery degradation and higher return for the end-user than with the single-objective optimisation. These differences compared to the single objective optimisation are due to fact that with MOTEEO the full Pareto front is considered when making the decision. In accordance with the weights presented in Table 6, the adopted solutions lead to higher overall benefits than the single-objective optimisations. Consistent with the previous results, cost minimisation with FFR provision is particularly adverse for the battery as the combination of Vehicleto-home (V2H) and V2G leads to a higher utilisation. As previously mentioned a lower energy cost leads inevitably to a higher grid impact and vice versa, because the price signal is not dynamically updated by to grid operator to better reflect the grid status. In addition, under the optimal grid net exchange, $\mathrm{CO}_{2}$ emissions are minimum. This effect will be particularly noticeable when the proposed methodology is applied to a real-life distribution system.

\subsection{Results of case study 2 for a distribution network}

\section{MOTEEO optimisation algorithm}

The application of MOTEEO for a typical UK DN allows the quantification of grid peak power and overall $\mathrm{CO}_{2}$ emissions at a higher scale compared to the single dwelling. Eight days have been simulated over the four seasons, including weekday and weekend. Four charging strategies, including uncontrolled charging (a), smart charging (b), bidirectional at home (c) and work (d) are simulated with two scenarios related to the ancillary service provision. Under uncontrolled charging, upon arrival the EVs are fully charged at the maximum power. Under smart charging, the charging process is controlled but the EVs are not discharged; hence under this strategy FFR cannot be provided. Bidirectional charging allow EVs to discharge towards the H-MG or the grid. Figure 9 and Figure 10 depict the MOTEEO scheduling for scenario $2 \mathrm{c}$ ) in the eight days. The preferred solution for the three decision makers, end-energy user, EV owners and DSO are shown. Other scenarios are not illustrated here for the purpose of conciseness. 

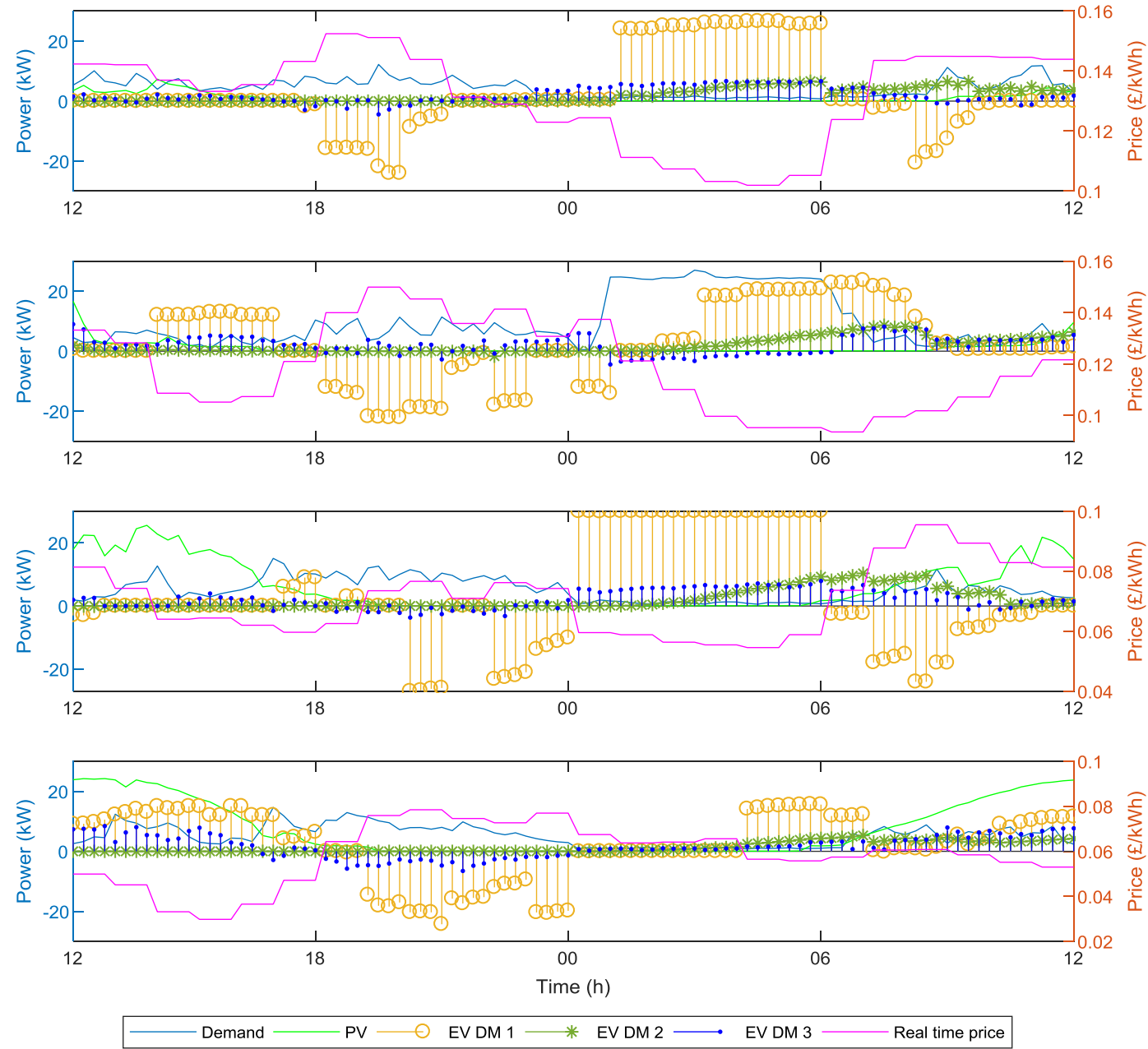

Figure 9 MOTEEO scheduling for scenario 2c) winter (weekday and weekend) and spring (weekday and weekend) from top to bottom 

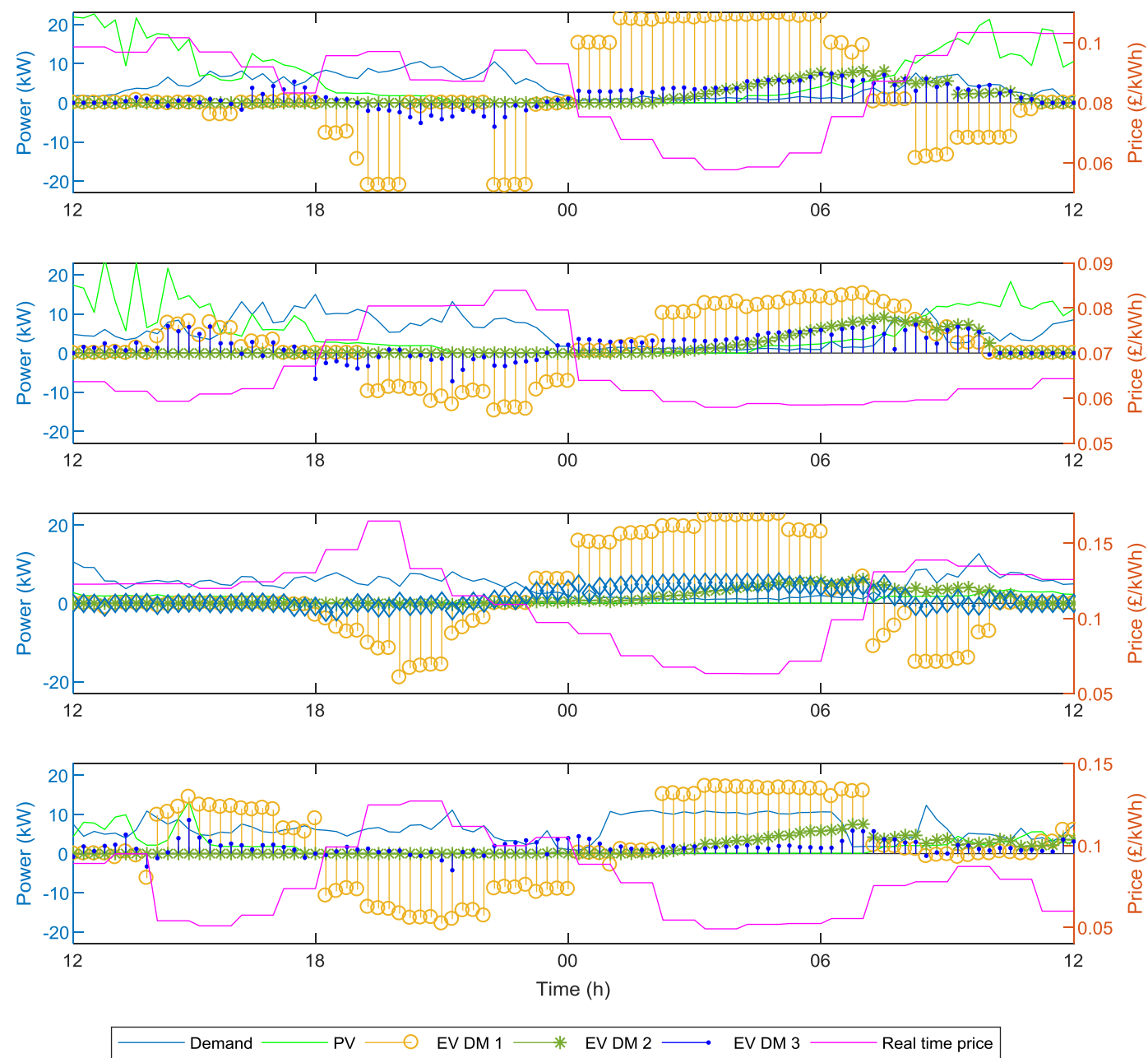

Figure 10 MOTEEO scheduling for scenario 2c) summer (weekday and weekend) and autumn (weekday and weekend) from top to bottom

As can be seen in Figure 9 and Figure 10, diverse PV generation and EV availability, have been simulated, due to seasonal effect and due to different travelling patterns on weekdays and weekends, respectively. As a general trend, EVs had higher availability for MOTEEO in the weekends. Higher availability and PV generation particularly benefitted grid net exchange minimisation under $\mathrm{DM}_{3}$, as can be seen in spring and summer weekends. Under $\mathrm{DM}_{2}$, to minimise battery degradation, the EVs are charged as close as possible to the respective departure times, while under $\mathrm{DM}_{1}$ price arbitrage is carried out.

Table 8 presents the results for case study 2. For all the scenarios, the MOTEEO framework calculated the Pareto fronts and the DMs chose the preferred solution based on the MCDM criteria outlined in section 3.8. As illustrating all the Pareto fronts generated in case study 2 would lengthen the paper we present the Pareto fronts for one day (summer weekend) in the appendix. 


\begin{tabular}{cccccc}
\hline Case & DM & $\begin{array}{c}\text { Energy } \\
\text { cost (f) }\end{array}$ & $\begin{array}{c}\text { Battery } \\
\text { degradation }(\mathfrak{f})\end{array}$ & $\begin{array}{c}\text { Grid net } \\
\text { exchange }(\mathbf{k W h})\end{array}$ & $\begin{array}{c}\text { Emissions } \\
\left(\mathbf{k g C O} \mathbf{O}_{\mathbf{2}}\right)\end{array}$ \\
\hline $\mathbf{2 a} \boldsymbol{i})$ & & -105.74 & -28.55 & 1917 & 489.88 \\
\hline \multirow{2}{*}{$\mathbf{2 b} \boldsymbol{i})$} & $\mathrm{DM}_{1}$ & -66.15 & -9.97 & 178 & 334.48 \\
& $\mathrm{DM}_{2}$ & -72.08 & -9.42 & 176.74 & 333.96 \\
& $\mathrm{DM}_{3}$ & -72.5 & -10.2 & 170.53 & 322.30 \\
\hline \multirow{2}{*}{$\mathbf{2 c} \boldsymbol{i})$} & $\mathrm{DM}_{1}$ & -52.79 & -50.01 & 299 & 481.91 \\
& $\mathrm{DM}_{2}$ & -71.40 & -9.52 & 176.72 & 333.69 \\
& $\mathrm{DM}_{3}$ & -72.80 & -20.39 & 174.07 & 322.65 \\
\hline \multirow{2}{*}{$\mathbf{2 d} \boldsymbol{i})$} & $\mathrm{DM}_{1}$ & -52.73 & -51.06 & 291.19 & 486.77 \\
& $\mathrm{DM}_{2}$ & -71.26 & -9.48 & 176.72 & 333.82 \\
& $\mathrm{DM}_{3}$ & -72.57 & -10.26 & 173.90 & 322.87 \\
\hline \multirow{2}{*}{$\mathbf{2 e}$ ii) } & $\mathrm{DM}_{1}$ & -12.38 & -26.74 & 276.1 & 392.50 \\
& $\mathrm{DM}_{2}$ & -20.83 & -13.59 & 176.32 & 342.03 \\
& $\mathrm{DM}_{3}$ & -22.18 & -17.5 & 171.26 & 334 \\
\hline
\end{tabular}

Under uncontrolled charging, the EVs are charged at maximum power until $80 \%$ of SOC is reached. This produces the highest values for all the metrics in Table 8, indicating that it is the worst scenario under all the criteria. The three rows for each case shows the results of the solution chosen by the three DMs, namely, end-electricity user, EV owners and $\mathrm{DSO}\left(D M_{1}, D M_{2}\right.$ and $D M_{3}$ respectively). With smart charging the battery degradation is kept to a minimum and there is little difference between the three DMs along this dimension. When bidirectional charging is employed, the improvements are higher; especially the energy cost can be further minimised as price arbitrage is performed. It should be pointed out that the performance along grid utilisation depends on the availability of PV generation (in colder months the performance is worse than the warmer months) and EV availability pattern; the EVs may not be available or at high SOC, therefore it would be unable to charge from PV. The different interests of the stakeholders are again evident: in 2c i), under the solution preferred by the DSO $\left(D M_{3}\right)$, the total utilisation is reduced from $291.19 \mathrm{kWh}$ to $173.9 \mathrm{kWh}(-40.28 \%)$ when compared with the solution chosen by the end-electricity. Conversely, this solution increases battery degradation compared to the solution chosen by the EV owners (increases degradation by 7.6\%). $\mathrm{CO}_{2}$ emissions are always at their minimum under the scheduling preferred by $D M_{3}$ as it utilises more local PV generation. Depending on the electricity demand profile of the dwellings and EV travelling pattern, this may not necessarily lead to the best solution along the other directions; this is because the EVs may be travelling when the peak demand occurs. When FFR is provided, it leads to an increase of all the metrics a part from the cost, because the optimisation window is reduced. However, FFR proves to be an overall profitable service as profits (£54.53) are higher than the incurred battery degradation cost (£3.53). Although an early replacement of the EV batteries may cause distress for the EV owners, this is taken into account by the battery degradation cost, which is offset by the prospective profits by a large margin (more than four times). As discussed in section 3.2, the cost of lithium-ion batteries is expected to drop in future, 
providing a better economic case. The forecasts predict a range of scenarios, where the average trend shows a cost reduction of $33 \%$ compared to current values. Despite the uncertainty in the future battery cost, any cost reduction will proportionally reduce the cost of battery degradation (battery purchase cost is included in the model). The positive consequence is that the use of EV batteries for energy services will become more cost-effective, which will improve the profitability of V2X services for all the stakeholders. A clear trade-off between the objectives is seen; the involved stakeholders must collaboratively take decisions and share benefits. It implies that all stakeholders must be sufficiently informed and capable of making informed decisions. Furthermore, a societal discussion will be required to see who can reap most of the benefits, and who must shoulder the burdens. To this end, the DSO is particularly suitable to manage this as a considerable improvement in grid utilisation is achieved which will defer grid investments. Therefore, it is in the DSO's interest to share the profit with the electricity users (in the form of reduced electricity bills), who lose $27.34 \%$ under the case chosen by $\mathrm{DM}_{3}$, and EV owners (subsidising part of their batteries), who lose 7.6\%, to stimulate participation to the MOTEEO program. If the profit is not shared, then end users and EV owners will not participate in delivering energy services and no peak reduction will be achieved; in the worst case, uncontrolled charging will cause negative impacts with increased EV penetration, and hence costs to the DSO.

\subsection{Results of case study 3 for cross-case comparison}

Smart incentives and intelligent tariff structures are critical for an effective implementation of MOTEEO. Among the possible solutions, the implementation of peak demand charges from the DSO, subsidy for the EV batteries and dynamic pricing are noteworthy. As an example of a smart tariff scheme, the case of commercial users in Flanders, Belgium is presented. Commercial users can purchase energy from the wholesale market but are charged transmission and distribution tariffs based on the peak demand [58]-[59]. We adapted this tariff to Case 1 scenario i) (without FFR) to highlight the importance of intelligent tariff schemes by applying the utility function [60] to combine the objectives with the value/cost they bring. The energy cost, the battery degradation cost and the peak demand charge have been combined in one function. Table 9 presents the result of Case 3.

Table 9 Results of the MOTEEO method with a utility function for one day

\begin{tabular}{ccccc}
\hline Energy cost (£) & $\begin{array}{c}\text { Battery } \\
\text { degradation }(\mathfrak{f})\end{array}$ & $\begin{array}{c}\text { Grid net exchange } \\
(\mathbf{k W h})\end{array}$ & $\begin{array}{c}\text { Emissions } \\
\left(\mathbf{k C O}_{\mathbf{2}}\right)\end{array}$ \\
\hline Case 3 & 0.14 & -0.0121 & 10.26 & 1.55 \\
\hline
\end{tabular}

By comparing Table 9 with the results of the single optimization in Table 5, a general improvement along all the dimensions can be seen. In fact, under this case, the peak demands are targeted, leading to a better performance along $\mathrm{Obj}_{3}$ and $\mathrm{Obj}_{4}$ but with a $28.1 \%$ reduction in battery degradation ( $(0.0121$ instead of $£ 0.0170$ ) when compared to the single-objective optimization of $O b j_{3}$. Therefore, the effectiveness of the utility function, which requires the cooperation of the three main stakeholders, has 
been demonstrated. Unfortunately, it is not always possible to assign a utility weight to all the objectives. Especially for $\mathrm{Obj}_{3}$, the peak demand penalty should be decided by the DSO in relation to the incurred investments for grid reinforcement, which should be calculated on a case-by-case basis (as these are both network and location specific). In countries/regions where these types of tariffs are not available, a joint decision between the involved stakeholders is critical to satisfy all the criteria.

\section{Conclusions}

In the present work, the MOTEEO approach is proposed and applied in three case studies and for different charging strategies in order to find the synergy of four objectives: energy cost, EV battery degradation, grid net exchange and $\mathrm{CO}_{2}$ emissions along with ancillary services. Mathematical models of the objectives and scenarios are constructed to represent the interests of the associated stakeholders. The conflicting objectives of stakeholders are resolved by multi-objective optimization with multicriteria-decision-making technique. By implementing the proposed methodology to the three case studies considered in this work some noteworthy conclusions were drawn and are summarised as follows.

Smart charging restricts battery degradation; however, it does not provide satisfactory cost improvement, grid and environmental impact minimization. The benefits of bidirectional charging are considerably higher (there is a revenue instead of a cost in terms of energy expense and $11 \%$ reduction in demand peak) compared to smart charging if EVs should be available during the day as well. Therefore, it is important that bidirectional chargers (and PV generation) are available at different locations, including work places. In addition, since battery degradation limits the performance along the other objectives, batteries should be operated under optimal conditions as much as possible (low temperature, SOC and charging rates). This could reduce battery degradation by $66.6 \%$ when compared to uncontrolled charging. Confirming previous work in literature, uncontrolled EV charging is the worst approach under all perspectives.

Under MOTEEO, the end-electricity users can increase their benefits by $81 \%$ (compare $2 \mathrm{e}$ with $2 \mathrm{~b}$ ) by providing frequency regulations service and the DSO can improve the grid utilisation by nearly $42 \%$. However, these are the maximum achievable benefits along one objective only, and there needs to be cooperation between the stakeholders to increase the overall social benefits. This suggests that a larger (or new) regulatory role must be played to ensure that overall social benefits are obtained. The DSO must share the benefits achieved from improved grid utilisation (investment cost deferral) by ensuring a revenue to the end-electricity user and the EV owner. The quantification of such revenue is casedependent and each distribution network should be studied individually. Therefore, a collaborative decision process has been proposed. The implementation of a smart utility function under MOTEEO targets the peak demand by combining the objectives of the end-electricity user and the DSO achieving optimal grid operation while minimizing the damage to the battery $(28.1 \%$ of reduction in battery degradation compared to the case without MOTEEO). This paper has demonstrated that a holistic 
decision-making process under MOTEEO is required, as not doing so will inevitably result in suboptimal consequences for other stakeholders and in the longer term, affect the social licence of that stakeholder and/or technology. Moreover, the MOTEEO framework allows costs and benefits to be quantified and discussed by the various stakeholders. The application of this framework in future energy systems will engage multiple stakeholders, increasing the utilisation of renewable energy sources and integrating the energy and transportation system. The cooperation among stakeholders through a decision-making process, as the one proposed in this paper, will bring overall societal benefits in future smart grids. The strategies proposed in this work optimise the utilisation of distributed energy resources, such as renewables and EVs, thus improving sustainability of the future energy landscape.

\section{Acknowledgement}

This work is supported in part by EU - Interreg North Sea Region programme - Smart, clean Energy and Electric Vehicles for the City (SEEV4-City) project J-No.: 38-2-23-15. The authors thank Prof. Krishna Busawon for his contribution to this research.

\section{Appendix}

If a PV installation is available, then assuming that the energy generation from the PV system cause zero-emission (life cycle $\mathrm{CO}_{2}$ emissions are out of scope for this paper) it follows that by minimizing grid net exchange $\mathrm{CO}_{2}$ emission is also minimised. If the PV installation is not available, then emissions are minimised if the $\mathrm{EV}$ discharges in periods with high specific $\mathrm{CO}_{2} / \mathrm{kWh}$. In the present work, we assume that a PV system is available. Figure 11 shows the result of the ANEC method minimizing both $\mathrm{CO}_{2}$ emission and grid net exchange. Given the negligible variation of the two objective functions among the 11 Pareto optimal solutions, we consider grid net exchange and $\mathrm{CO}_{2}$ emission minimisation to be equivalent.

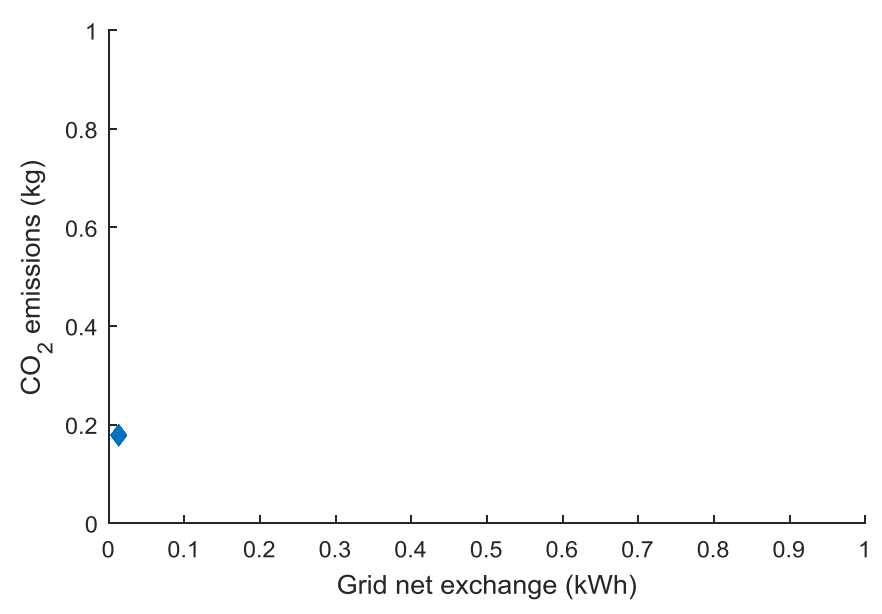

Figure 11 Bi-objective optimisation of $\mathrm{CO}_{2}$ emissions and grid net exchange 
Figure 12 shows the normalised Pareto fronts (with respect to the maximum values of the objectives) for 10 EVs under scenario 2c) bidirectional charging for a summer weekend. The conflict among the different objectives is noticeable as minimising one objective reduces the performance along other objectives.

EV1
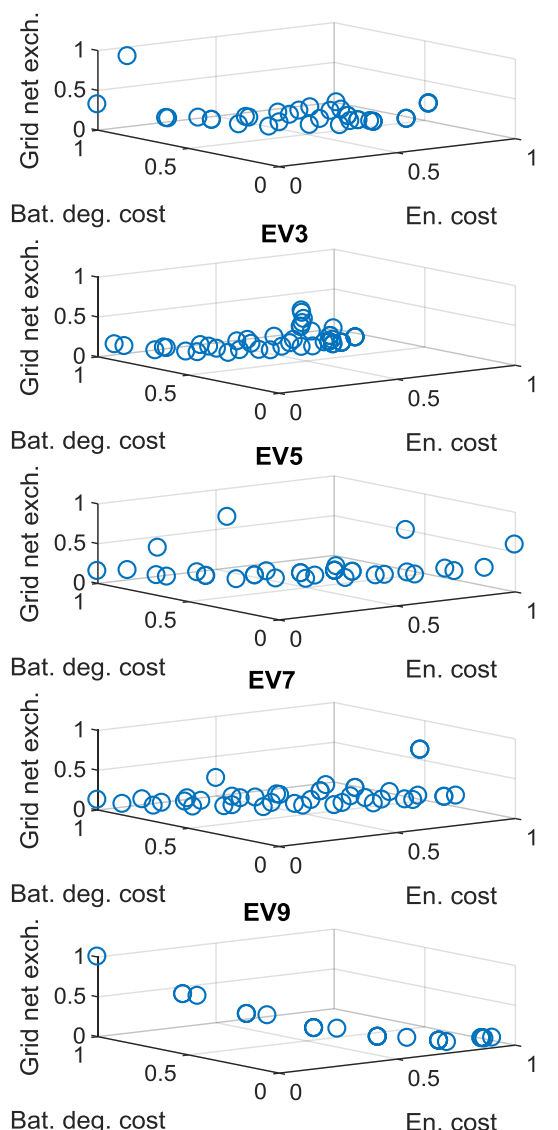

Bat. deg. cost
En. cost

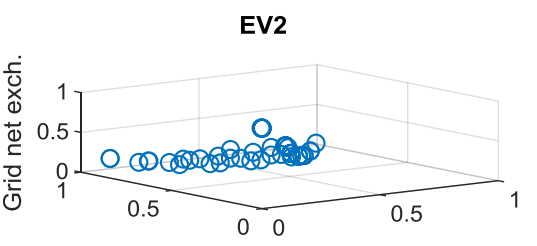

Bat. deg. cost EV4 En. cost
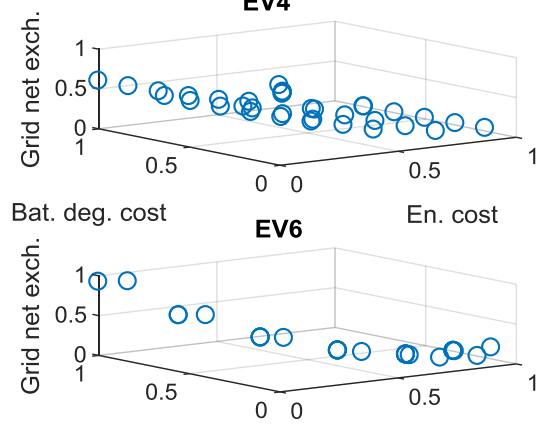

Bat. deg. cost $\quad$ En. cost

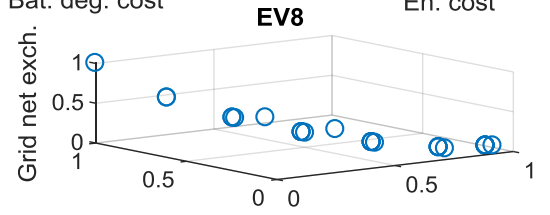

Bat. deg. cost EV $\quad$ En. cost

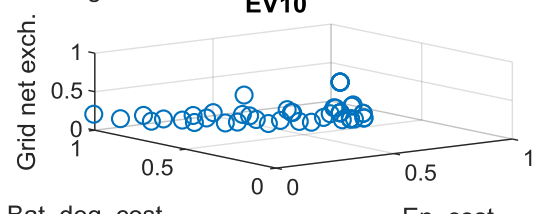

Bat. deg. cost

En. cost

Figure 12 Pareto fronts for 10 EVs, under scenario 2c) in a summer weekend

\section{References}

[1] Global EV Outlook 2018 - Towards cross-modal electrification, International Energy Agency, Clean Energy Ministerial, France, 2018.

[2] A. Schuller, B. Dietx, C. M. Flath, C. Weinhardt, Charging Strategies for Battery Electric Vehicles Economic Benchmark and V2G Potential, IEEE Transactions on Power Systems. Vol. 29, No. 5, pp. 214-222, September 2014.

[3] T. Ma, O. A. Mohammed, Economic Analysis of Real-Time Large-Scale PEVs Network Power Flow Control Algorithm With the Consideration of V2G Services, IEEE Transactions on Industry Applications, Vol. 50, No. 6, November/December 2014. 
[4] M. J. E. Alam, K. M. Muttaqi and D. Sutanto, Effective Utilisation of Available PEV Battery Capacity for Mitigation of Solar PV Impact and Grid Support With Integrated V2G Functionality, in IEEE Transactions on Smart Grid, Vol. 7, No. 3, pp. 1562-1571, May 2016.

[5] R. Lamedica, S. Teodori, G. Carbone and E. Santini, An energy management software for smart buildings with V2G and BESS”, Sustainable Cities and Society, Vol. 19, pp. 173-183, 2015.

[6] K. N. Kumar, B. Sivaneasan, P. H. Cheah, P. L. So and D. Z. W. Wang, V2G Capacity Estimation Using Dynamic EV Scheduling, in IEEE Transactions on Smart Grid, Vol. 5, No. 2, pp. 10511060, March 2014.

[7] E. Xydas, C. Marmaras, L. M. Cipcigan, A multi-agent based scheduling algorithm for adaptive electric vehicles charging, Applied Energy, Vol. 177, pp. 354-365, 2016.

[8] H. Xing, M. Fu, Z. Lin and Y. Mou, Decentralised Optimal Scheduling for Charging and Discharging of Plug-In Electric Vehicles in Smart Grids, in IEEE Transactions on Power Systems, Vol. 31, no. 5, pp. 4118-4127, Sept. 2016.

[9] G. Binetti, A. Davoudi, D. Naso, B. Turchiano and F. L. Lewis, Scalable Real-Time Electric Vehicles Charging With Discrete Charging Rates, in IEEE Transactions on Smart Grid, Vol. 6, no. 5, pp. 2211-2220, Sept. 2015.

[10] H. N. T. Nguyen, C. Zhang and M. A. Mahmud, Optimal Coordination of G2V and V2G to Support Power Grids With High Penetration of Renewable Energy, in IEEE Transactions on Transportation Electrification, Vol. 1, no. 2, pp. 188-195, Aug. 2015.

[11] L. Drude, L. C. Pereira Junior and R. Rüther, PhotoVoltaics (PV) and electric vehicle-to-grid (V2G) strategies for peak demand reduction in urban regions in Brazil in a smart grid environment, Renewable Energy, Vol. 68, pp. 443-451, 2014.

[12] M. Tavakoli, F. Shokridehaki, M. Marzband, R. Godina and E. Pouresmaeil, A two stage hierarchical control approach for the optimal energy management in commercial building microgrids based on local wind power and PEVs, Sustainable Cities and Society, Vol. 41, pp. 33234, 2018.

[13] K. M. Tan, V. K. Ramachandaramurthy and J. Ying Yong, Optimal vehicle to grid planning and scheduling using double layer multi-objective algorithm, Energy, Vol. 112, pp. 1060-1073, 2016.

[14] W. Yao, J. Zhao, F. Wen, Z. Deng, Y. Xue, Y. Xu, and K. Meng, A Multi-Objective Collaborative Planning Strategy for Integrated Power Distribution and Electric Vehicle Charging Systems, in IEEE Transactions on Power Systems, Vol. 29, no. 4, pp. 1811-1821, July 2014.

[15] S. S. Amiri, S. Jadid and H. Saboori, Multi-objective optimum charging management of electric vehicles through battery swapping stations, Energy, Vol. 165, pp. 549-562, 2018.

[16] J. Li and M. A. Danzer, Optimal charge control strategies for stationary photoVoltaic battery systems, Journal of Power Sources, Vol. 258, pp. 365-373, 2014.

[17] A. Zakariazadeh, S. Jadid and P. Siano, Multi-objective scheduling of electric vehicles in smart distribution system, Energy Conversion and Management, Vol. 79, pp 43-53, 2014. 
[18] M. J. Morshed, J. B. Hmida and A. Fekih, A probabilistic multi-objective approach for power flow optimization in hybrid wind-PV-PEV systems, Applied Energy, Vol. 211, pp. 1136-1149, February 2018.

[19] J. Li, X. Jin and R. Xiong, Multi-objective optimization study of energy management strategy and economic analysis for a range-extended electric bus, Applied Energy, Vol. 194, pp. 798-807, 2017.

[20] J. García-Villalobos, I. Zamora, K. Knezovic' and M. Marinelli, Multi-objective optimization control of plug-in electric vehicles in low Voltage distribution networks, Applied Energy, pp. 155$168,2016$.

[21] M. Marzband, M. Javedi, S. A. Pourmousavi and G. Lightbody, An advanced retail electricity market for active distribution systems and home microgrid interoperability based on game theory, Electric Power Systems Research, Vol. 157, pp 187-199, 2018.

[22] G. Platt, The Decentralised Control of Electricity Networks Intelligent and Self-Healing Systems, 2007.

[23] Y. Zhou, J. Wu and C. Long, Evaluation of peer-to-peer energy sharing mechanisms based on a multiagent simulation framework", Applied Energy, Vol. 222, pp. 993-1022, 2018.

[24] A. Baliyan, K. Gaurav and S. Kumar Mishra, A Review of Short Term Load Forecasting using Artificial Neural Network Models, Procedia Computer Science, Vol. 48, 2015, pp. 121-125.

[25] https://www.ovoenergy.com/electric-cars/vehicle-to-grid-charger

[26] https://nuvve.com/

[27] https://innovation.ukpowernetworks.co.uk/projects/transpower-vehicle-to-grid/

[28] K. Uddin, T. Jackson, W. D. Widanage, G. Chouchelamane, P. A. Jennings, J. Marco, On the possibility of extending the lifetime of lithium-ion batteries through optimal V2G facilitated by an integrated vehicle and smart-grid system, Energy, Vol. 133, pp. 710-722, 2017.

[29] Element Energy, Cost and performance of EV batteries, March 2012.

[30] C. Curry, Lithium-ion Battery Costs and Market, Bloomberg New Energy Finance

[31] G. Berckmans, M. Messagie, J. Smekens, N. Omar, L. Vanhaverbeke and J. Van Mierlo, Cost Projection of State of the Art Lithium-Ion Batteries for Electric Vehicles Up to 2030, Energies, September 2017.

[32] R. Kochhan, S. Fuchs, B. Reuter, P. Burda, S. Matz and M. Lienkamp, An Overview of Costs for Vehicle Components, Fuels and Greenhouse Gas Emissions, Institute of Automotive Technology, 2014.

[33] N. Narayan, T. Papakosta, V. Vega-Garita, Z. Qin, J. Popovic-Gerber, P. Bauer and M. Zeman, Estimating battery lifetimes in Solar Home System design using a practical modelling methodology, Applied Energy, Vol. 228, pp.1629-1639, 2018.

[34] G. Lacey, G. Putrus and E. Bentley, Smart EV charging schedules: supporting the grid and protecting battery life, in IET Electrical Systems in Transportation, Vol. 7, no. 1, pp. 84-91, 32017. 
[35] M. Dubarry, A. Devie and K. McKenzie, Durability and reliability of electric vehicle batteries under electric utility grid operations: Bidirectional charging impact analysis, Journal of Power Sources, Vol. 358, pp. 39-49, 2017.

[36] G. Lacey, Evaluation of Lithium Ion Battery Degradation and the Implications for the Use of EV batteries in Providing Support to a Smart Grid, a Doctoral thesis, Northumbria University, March 2015.

[37] J. Tomić and W. Kempton, Using fleets of electric-drive vehicles for grid support, Journal of Power Sources, Vol. 168, pp.459-468, 2007.

[38] C-H. Hwang and AS.M. Masud, Abu, Multiple Objective Decision Making-Methods and Applications: A State-of-the-Art Survey, 1979, 10.1007/978-3-642-45511-7.

[39] R. Mehri and M. Kalantar, Multi-objective scheduling of electric vehicles considering wind and demand uncertainties, 2015 Smart Grid Conference (SGC), Tehran, pp. 122-129, 2015.

[40] C. Deng, N. Liang, J. Tan and G. Wang, Multi-Objective Scheduling of Electric Vehicles in Smart Distribution Network, Sustainability, November 2016.

[41] A. Zakariazadeh, S. Jadid and P. Siano, Multi-objective scheduling of electric vehicles in smart distribution system, Energy Conversion and Management, Vol. 79, 2014, pp 43-53,

[42] K. Knezovic, A. Soroudi, M. Marinelli, and A. Keane, Robust Multi-Objective PQ Scheduling for Electric Vehicles in Flexible Unbalanced Distribution Grids, IET Generation Transmission and Distribution, 11(16), pp. 4031-4040, 2017.

[43] G. Mavrotas, Effective implementation of the $\varepsilon$-constraint method in Multi-Objective Mathematical Programming problems, Applied Mathematics and Computation, Vol. 213, pp. 455465, 2009.

[44] R.W. Saaty, The analytic hierarchy process - what it is and how it is used, Mathematical Modelling, Vol. 9, pp. 161-176, 1987.

[45] J. M. Hummel, J. F. P. Bridges and M. J. Ijzerman, Group Decision Making with the Analytic Hierarchy Process in Benefit-Risk Assessment: A Tutorial, The Patient - Patient-Centered Outcomes Research, Vol. 7, pp. 129-140, 2014.

[46] https://www.ofgem.gov.uk/data-portal/breakdown-electricity-bill

[47] G. A. Putrus, P. Suwanapingkarl, D. Johnston, E. C. Bentley and M. Narayana, Impact of electric vehicles on power distribution networks, 2009 IEEE Vehicle Power and Propulsion Conference, Dearborn, MI, pp. 827-831, 2009.

[48] M. Nicoli, R. Das, Y. Wang, G. Putrus and R. Kotter, A Smart Grid Modelling Tool for Evaluating Optimal Control of Electric Vehicles, 53rd International Universities Power Engineering Conference (UPEC), Glasgow, 2018.

[49] Y. Wang and D. Infield, Markov Chain Monte Carlo simulation of electric vehicle use for network integration studies, International Journal of Electrical Power \& Energy Systems, Vol. 99, pp. 8594, 2018. 
[50] E. McKenna and M. Thomson, High-resolution stochastic integrated thermal-electrical domestic demand model, Applied Energy, Vol. 165, pp. 445-461, 2016.

[51] National Grid, Future Energy Scenarios, July 2018.

[52] https://www.ft.com/content/30f7e328-8372-11e8-96dd-fa565ec55929

[53] https://www.gov.uk/government/statistics/solar-photoVoltaics-deployment

[54] https://www.gov.uk/government/statistics/energy-consumption-in-the-uk

[55] A. Evans, J. Commings, M. Slocombe and F. Corvaglia, National Travel Survey: England 2017, Department for Transport, July 2018. Accessed online 28/01/2019. Available at (https://assets.publishing.service.gov.uk/government/uploads/system/uploads/attachment data/fil e/729521/national-travel-survey-2017.pdf).

[56] Department of Transport statistics, National Travel Survey. Accessed online 28/01/2019. Available at (https://www.gov.uk/government/statistics/national-travel-survey-2017).

[57] FFR pre and post tender. Accessed online 30/07/2019. Available at (https://www.nationalgrideso.com/balancing-services/frequency-response-services/firmfrequency-response-ffr?market-information)

[58] R. Cleenwerck, Potentieel van een smart grid om de energieautonomie van een stadsgebouw te verhogen: een case study in Kortrijk, a Masters Thesis, 2017.

[59] B. Rotthier, B. Derijcke, R. Leenders, N. Van den Steen, B. Huyck and J. Capelle, Comparison of the contribution of smart charging. V2G and energy demand reduction to the energy autonomy of a Belgian city depot, Electric Vehicle Symposium 31, October 2018.

[60] R. S. Walia, H. S. Shan and P. Kumar, Multi-Response Optimisation of CFAAFM Process Through Taguchi Method and Utility Concept, Materials and Manufacturing Processes, Vol. 21, pp. 907-914, 2006. 